\title{
Coherence Imaging Spectroscopy at Wendelstein 7-X for impurity flow measurements
}

Valeria Perseo, ${ }^{1}$ | Dorothea Gradic, ${ }^{1}$ Ralf König, ${ }^{1}$ Oliver P. Ford,${ }^{1}$ Carsten Killer, ${ }^{1}$ Olaf Grulke, ${ }^{1}$ and David A. Ennis ${ }^{2}$ (the W7-X Team) ${ }^{\dagger}$

\author{
${ }^{1}$ Max-Planck-Institut für Plasmaphysik, 17491 Greifswald, Germany \\ ${ }^{2}$ Department of Physics, Auburn University, 36849 Auburn, Alabama, USA
}

(Dated: January 16, 2020)

\begin{abstract}
In the last decade, Coherence Imaging Spectroscopy (CIS) has shown distinctive results in measuring ion flow velocities in the edge of magnetically confined plasma devices. Its $2 \mathrm{D}$ spatially resolved measurement capabilities and its high optical throughput are ideal for investigating the impurity behavior in the complex 3D magnetic island topology edge of Wendelstein 7-X (W7-X). However, a highly precise and stable calibration method is required for a reliable diagnostic operation. A new level of precision and stability has been achieved for the two CIS systems installed at W7-X with the use of a new calibration source, a continuous tunable laser commercially available only since 2015. A specific prototype model was successfully adapted to the challenging requirements of W7-X, granting high accuracy $( \pm 0.01 \mathrm{pm})$ and flexibility (spectral range: $450-650 \mathrm{~nm})$ in the wavelength calibration required for measuring low-Z impurity ion flow velocities. These features opened up new investigation possibilities on temperature stability and wavelength response of the CIS components, allowing to fully characterize and validate the W7-X systems. The CIS diagnostic was operational throughout the last W7-X experimental campaign. Measured velocities on the order of $\sim 20-30 \mathrm{~km} / \mathrm{s}$ were observed, corroborated by comparisons to measurements with Mach probes.
\end{abstract}

Keywords: coherence imaging spectroscopy, stellarator, impurity flows, calibration

* Correspoding author; e-mail: valeria.perseo (at) ipp.mpg.de

${ }^{\dagger}$ For the complete member list, please refer to T. Klinger et al., Nuclear Fusion 59, 112004 (2019) 


\section{INTRODUCTION}

In magnetic fusion devices, studying the scrape-off layer (SOL) dynamics is crucial for understanding the divertor exhaust concept. In this context, flow measurements, in combination with dedicated simulations, are essential to understand the physics behind energy, particle, and momentum transport. Important results have been already

5 achieved by Mach probes and traditional spectroscopy [1 4. Nonetheless, these diagnostics present some constraints, such as power handling ability, time and flow velocity resolution, or the limited number of lines of sight.

Most of these limitations can be overcome by using the Coherence Imaging Spectroscopy (CIS) diagnostic. After its invention nearly three decades ago [5], different techniques have been developed in order to achieve the measurement of 2D Doppler line shifts on the order of picometers [6]. The most recent applications are camera-based heterodyne 10 polarization interferometers, able to detect wavelength variations for spectral line emission in the visible range. This CIS technique superimposes an interference pattern on the plasma image by the use of birefringent crystals sandwiched between two polarizers 6 8) (Fig. 1). The spectral line shift and broadening are recovered with a 2D (spatial) Fourier analysis. For the diagnostic to function correctly, it is important that spectral lines from only one charged state of one species are selected from the total visible plasma spectrum by a bandpass filter. Since the

15 spectroscopic capabilities of CIS do not depend on the utilization of slits, the diagnostic is characterized by a high optical throughput, allowing improved time resolution with respect to traditional ultra-high-resolution spectroscopy $(<20 \mathrm{pm} / \mathrm{pixel})$. CIS diagnostics have been used on many fusion experiments, including MAST [9], DIII-D [10 12], and ASDEX Upgrade [13.

Two CIS systems were recently installed on W7-X, in order to observe the stellarator divertor region from nearly 20 perpendicular views [14]. The systems have been optimized in order to measure impurity flows and were extensively used during the last experimental campaign OP1.2 (2017-2018), with focus on C III emission (at $464.8811 \mathrm{~nm}$ ) from the SOL.

In order to perform reliable low-Z impurity flow measurements, a high wavelength calibration accuracy is required, which is guaranteed at W7-X by the implementation of a new calibration source, a continuous tunable laser. Other

25 recent laser calibration techniques have already shown improved results with respect to standard spectral lamp applications [15. However, these implementations are based on laser diodes, which have constraints in temperature stability, tuning capabilities and wavelength availability. These limitations are overcome with the W7-X laser, as it stands out for its fine (software) tuning capability $\left(10^{-2} \mathrm{pm}\right)$ in a wide wavelength range $(450-650 \mathrm{~nm})$, which allows to emulate the wavelength shift expected during a plasma discharge $(\sim 10 \mathrm{pm})$ and the exact, unshifted position of an

30 investigated line for many different reference wavelengths. This is critical in accurately calibrating both the direction and the zero point of the impurity flow without relying on simulations [16. This new level of accuracy, together with the 2D flow imaging capability of CIS, allowed to gain new information about the effects of the complex 3D geometry of W7-X. In particular, the counter-streaming flows induced by the overall low-shear magnetic island topology have been successfully measured and compared with dedicated EMC3-EIRENE numerical simulations for the first time [17.

35 Moreover, the behavior of the intrinsic impurities was observed under different plasma scenarios, e.g. with different input energy, main ion density, and magnetic configurations. The magnitude of the observed flow velocities varied significantly. The maximum detected was $35 \mathrm{~km} / \mathrm{s}$ with an error of $\pm 3 \mathrm{~km} / \mathrm{s}$.

This paper reports about the assembly and test of the CIS diagnostic setup at W7-X. The experimental setup is described in Sec II] followed by a discussion on the laser calibration and its applications in Sec.III. The CIS systems 40 have also been compared with Mach probes, as presented in Sec.IV.

\section{COHERENCE IMAGING SPECTROSCOPY SETUP}

As previously mentioned, two systems have been set up for W7-X (in the following designated as toroidal and vertical), which share the same designs for the main components.

A picture of one the two systems is shown in Fig 2. The labels will be used as a reference in the following explanation 45 of the components.

The plasma light is collected by $16 \mathrm{~mm}$ focal length lenses, each one connected to an image fiber bundle, which is composed of $800 \times 100010 \mu \mathrm{m}$ diameter fibers $(\mathrm{NA}=0.63$, by Schott). The equipment is placed inside immersion tubes (visible in Fig, 3), necessary due to the cryostat of W7-X [18. Each immersion tube ends with a sapphire window directly facing the plasma. For details on the immersion tubes design, please refer to [19, 20] for the toroidal

50 view (without pinhole) and to [21] for the vertical view. The use of the fiber bundles substantially decreases the amount of transmitted plasma light. Nonetheless, it allows the exploitation of hardly accessible diagnostic ports, the adoption of a manageable magnetic screening, and it avoids additional issues linked to thin film coating of in-vessel optical components [12. The maximum available length of the image bundle is $4.5 \mathrm{~m}$, thus the diagnostic needs to be placed close to W7-X. After the fiber bundle, a series of objective lenses have been mounted (Fig 2 no.2-3), chosen to 


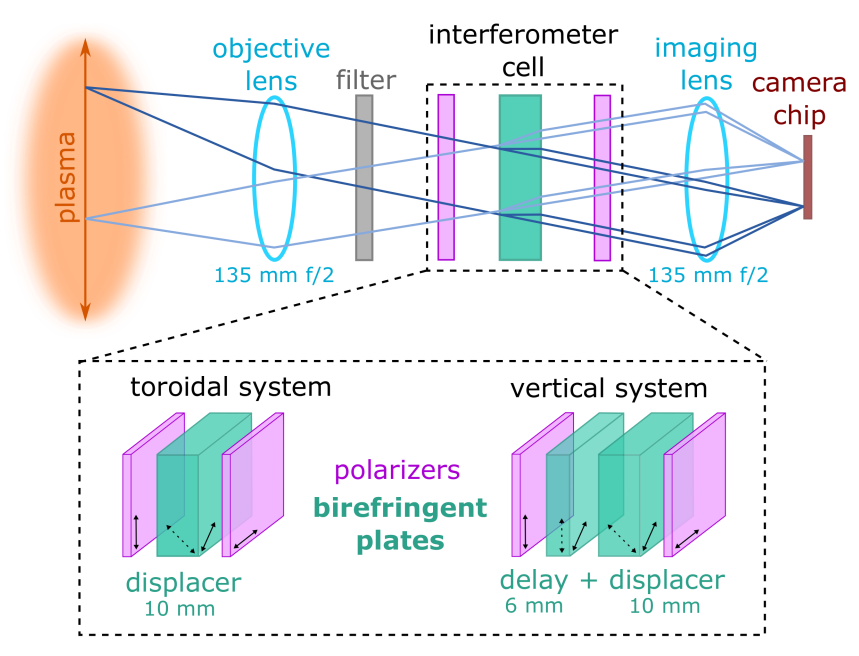

FIG. 1. Schematic of the Coherence Imaging Spectroscopy concept. The main components are represented in different colors. The solid black arrows indicate the direction of the optical axes of the polarizers and the birefringent crystals. For the latter, the projection of the optical axes on a second surface is displayed with dashed arrows in order to highlight the difference between delay and displacer plates.

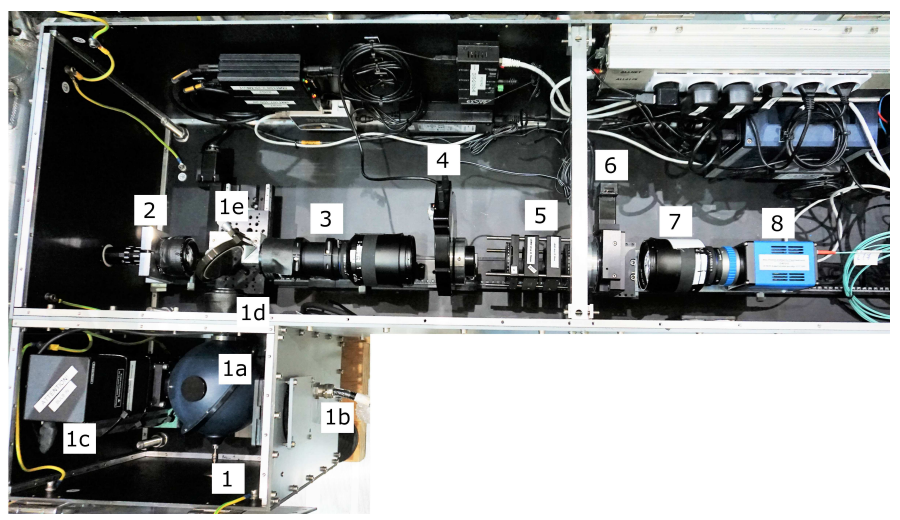

FIG. 2. Picture of the vertical CIS system at W7-X. Shown components: 1 - calibration system, of which 1a-integrating (Ulbricht) sphere, 1b-fiber for laser light, 1c-spectral lamp, 1d-objective lens ( $50 \mathrm{~mm})$, 1e-movable mirror; 2 - first lens (50 mm); 3-lens tandem $(50 \mathrm{~mm}$ and $135 \mathrm{~mm}) ; 4$ - filter wheel; 5-interferometer cell; 6-rotator; 7-imaging lens (135 mm); 8 - camera. The electronics, the optical rail, and the soft iron box are partially visible as well.

55 minimize, at the same time, vignetting effects and light divergence in the full system length. Table 1 contains detailed information about the used lenses. Interference filters with a $2 \mathrm{~nm}$ FWHM transmission width are used to select light of only one charge state of one plasma impurity.

The chosen cameras (Fig,2 no.8) are pco.edge 5.5 CLHS, characterized by a sCMOS sensor with $2560 \times 2160$ active pixels, $6.5 \mu \mathrm{m} \times 6.5 \mu \mathrm{m}$ each, i.e. the sensor area is $16.6 \mathrm{~mm} \times 14.0 \mathrm{~mm}$. The camera is capable of sustaining up to $60 \sim 0.5 \mathrm{~GB} / \mathrm{s}$ data streaming. The quantum efficiency is above $60 \%$ at peak and the maximum frame rate is $50 \mathrm{fps}$

\begin{tabular}{llll}
\hline \hline Lens name & Lens manufacturer & Focal length & f/\# \\
PFL - toroidal & Navitar & $16 \mathrm{~mm}$ & 1.4 \\
PFL - vertical & Schneider & $16 \mathrm{~mm}$ & 1.8 \\
$1 \mathrm{~d}, 2$, first of 3 & Nikon & $50 \mathrm{~mm}$ & 1.2 \\
second of 3,7 & Zeiss & $135 \mathrm{~mm}$ & 2 \\
\hline \hline
\end{tabular}

TABLE I. List of the used lenses in the two CIS systems setup. PFL here is intended as plasma facing lens. The numbers used as names refer to Fig 2 The lens setup is the same for the two systems, unless stated otherwise. 


\begin{tabular}{llcc}
\hline \hline & & Total \# & Achieved \\
System & Crystals & contrast \\
Toroidal & $10 \mathrm{~mm}$ displacer & 1383 & $88 \%$ \\
Vertical & $10 \mathrm{~mm}$ displacer & 2953 & $68 \%$ \\
& $+6 \mathrm{~mm}$ delay & & \\
\hline \hline
\end{tabular}

TABLE II. List of the chosen birefringent crystals setup for the two CIS systems. The total number of waves delay is calculated in the center of the image with equation (12) in [26] divided by $2 \pi$. The achieved contrast is here intended as the best value measured in calibration images.

in case of full chip readout in global shutter mode. The dynamic range is 27000:1 with a full well depth of 30000 photoelectron counts. The noise level stays below 143 electrons per pixel. With the chosen lenses setup, only $\sim 2 / 3$ of the chip has been used for recording. The set exposure time varies depending on plasma conditions, observed impurity, and magnetic configuration. In general, it was chosen in order to maintain around 2300 photoelectrons at 65 minimum in the divertor region. At this level, the signal-to-noise ratio leads to a statistical error on the evaluated velocity one order of magnitude below the $3 \mathrm{~km} / \mathrm{s}$ considered as systematic error. For the brightest impurity spectral lines (e.g. C III at $464.8811 \mathrm{~nm}$ ), the average chosen exposure time has been $60 \mathrm{~ms}$ for the toroidal system, and $90 \mathrm{~ms}$ for the vertical.

Due to the novelty of the CIS application on W7-X and the limited diagnostic accessibility during experimental 70 days, some additional motorized components, remotely controllable, are included in the design to increase the overall system flexibility. In particular, a filter wheel and a rotator attached to the interferometer cell (Fig,2 no.4-6) are introduced to change respectively the species under study and the direction of the interference fringes (i.e. of highest spatial resolution [22]).

The core part of the CIS diagnostic is the interferometer cell (Fig2, no.5), composed of two crossed polarizers placed 75 before and after a set of one or more fixed-delay birefringent plates with their optical axes at $45^{\circ}$ with respect to the orientation of the polarizers (Fig 11). Birefringence is used to generate interference between the two polarizations of light: the first polarizer ensures that the two polarizations of the incoming light are equal in amplitude, the plates introduce a phase between the two polarizations and split them, and the last polarizer puts the two polarizations back in one plane allowing interference. More specifically, the combination of the crystals creates an angle-dependent 80 interferometric phase shear, necessary to generate approximately parallel spatial interference fringes on the camera chip [6]. This fringe pattern acts as a spatial heterodyne carrier for Doppler amplitude and phase modulations that can be recovered using numerical Fourier demodulation techniques. More details on the diagnostic principles can be found elsewhere [10, 22. The standard arrangement of the birefringent plates is formed by a delay plate and a displacer plate. The difference between the two plates regards the direction of the optical axis of the crystals with 85 respect to their surfaces: the delay plate has its axis parallel to the surface, whereas the displacer plate has it at $45^{\circ}$ with respect to the crystal interface. A helpful but simplistic explanation of the practical implications is the analogy with the Michelson interferometer: the displacer plate acts like the beam splitter (spatially separating the two polarizations of the incoming light) and the delay plate acts like the optical path difference (introducing a phase between the two polarizations) [23. In reality, an additional phase is introduced by the displacer plate that has to be

90 taken into account in the choice of the crystals setup. Because of this, the system can also be composed of only a thick displacer plate, at the cost of a decreased flexibility. The choice for the plates setup depends on multiple variables to be optimized, i.e. the number of pixels per fringe, and the induced phase and contrast. The first two parameters, respectively linked to the CIS spatial and velocity resolution, can be improved with the use of thicker plates. On the other hand, the fringe contrast, defined as how distinguishable the interference pattern is over the plasma image, has a 95 non-trivial dependence on the plate thickness, as it is linked to the structure of the plasma spectral line to be observed 22]. At W7-X, two different interferometer cells have been chosen for the two systems, both optimized to measure the C III multiplet at $464.8811 \mathrm{~nm}$ : a $10 \mathrm{~mm}$ thick displacer plate for the toroidal one, and a combination of $10 \mathrm{~mm}$ thick displacer and $6 \mathrm{~mm}$ thick delay plates for the vertical one (as shown in Fig.1). The choice followed calculations based on the method explained in 22]: the selected crystals are in a local maximum in contrast, which also gives sufficient

100 delay for $\mathrm{C}$ III, He II, and $\mathrm{H}_{\beta}$ spectral lines. Having the displacer plate with the same thickness, both systems are characterized by $\sim 12$ pixels/fringe. The chosen material for the crystals is alpha-barium borate $\left(\alpha-\mathrm{BBO}, \mathrm{BaB}_{2} \mathrm{O}_{4}\right)$, due to its low sensitivity to temperature variations with respect to other common birefringent materials [23, 24]. The crystal setup did not show the necessity of using field widening techniques 25. The main plates parameters of the two systems are displayed in Table II The crystals are mounted in a cage system with removable segments, which 105 eases the exchange of different plate setups. The interferometer cell and the camera are mounted on the same support to minimize changes of their relative positions and therefore avoiding additional phase variations.

The systems are placed in soft iron boxes of $5 \mathrm{~mm}$ thickness positioned at roughly $1.5 \mathrm{~m}$ from the outer vessel of 


\begin{aligned} & \hline \hline Wavelength range $450-525 \mathrm{~nm}$ and $540-650 \mathrm{~nm} \\ &$ Wavelength accuracy $0.01 \mathrm{pm} \\ &$ Wavelength stability $\leq 5 \mathrm{MHz}$ over $10 \mathrm{~h} \\ &$ Linewidth $<1 \mathrm{MHz}$, typical $<500 \mathrm{kHz} \\ &$ Output power (VIS) min $>80 \mathrm{~mW}$, typical $>150 \mathrm{~mW} \\ &$ Beam radius $0.5 \mathrm{~mm} \\ &$\hline \hline\end{aligned}

TABLE III. List of the C-Wave laser features.

W7-X, thereby reducing the magnetic field produced by the superconducting coils of the machine by $\sim 95 \%$, getting to 3-4 mT. Since the magnetic field of W7-X usually remains constant throughout an experimental day, none of the 110 effects shown in [12] have been observed. All optical components are mounted on a rigid optical rail and the soft iron boxes are fixed on scaffolds, in order to minimize the effect of vibrations. One of the two systems, the toroidal one, is sharing the soft iron box and the lines of sight with a fast video camera system. The electric power is provided via remotely controllable power strips, which are equipped with a sensor able to record temperature and humidity every five minutes.

115 The main difference between the W7-X and other CIS designs is the calibration system. For Doppler velocity measurements, it is essential to be able to use the wavelength emission of the particle at rest as a reference $\left(\lambda_{0}\right)$. The high velocity resolution, typical of CIS, sets a demanding accuracy level in order to have a reliable calibration. At W7$\mathrm{X}$, the high degree of accuracy is reached by using a continuous tunable laser (C-Wave, by Hübner photonics) [16]. The C-Wave emission comes from a diode-pumped solid-state laser (Nd:YAG, emitting at $532 \mathrm{~nm}$ ) that passes through two 120 cavities containing lithium niobate $\left(\mathrm{LiNbO}_{3}\right)$ crystals: a Optical Parametric Oscillator (OPO) and a Second-Harmonic Generator (SHG), both characterized by non-linear optical behaviors. The OPO is responsible for the tuning process. In this cavity, the incoming photon is split into two photons of lower energy, therefore the tuning process happens in infra-red light. In order to get back into the visible range, the SHG is used, a cavity that simply doubles the frequency of the incoming light. The crystal temperature and the cavity length are the main parameter that can be changed

125 27. For the W7-X CIS application, a customized prototype has been produced due to demanding requirements. In particular, the required wavelength accuracy and stability need additional control over the tuning process: the OPO infra-red output is monitored and steered in a feedback loop with a wavemeter (HighFinesse WS7/30) by a dedicated software called AbsoluteLambda. This method grants, for the first time, tuning steps of 0.05 pm and long term stability in the same order of magnitude, over almost the entire visible range. Moreover, a remotely controlled

130 shutter has been introduced in order to handle when the laser light is entering the CIS systems. The C-Wave main features are listed in Table III. The C-Wave laser has been placed outside the torus hall and its light is carried to the diagnostics with $\sim 80 \mathrm{~m}$ long multi-mode fibers. The fibers are connected to speckle reducers (Optotune LSR 3005) mounted on the entrance port of the integrating (Ulbricht) spheres, in order to obtain a homogeneous calibration emission output and to avoid problems related to the laser long coherence length. The light is introduced in the

135 CIS system with an imaging lens and an additional optical component (Fig,2, no.1a-e), that is a beamsplitter for the toroidal system and a movable mirror for the vertical one. The objective lens at the sphere (Fig,2, no.1d) is of the same type as the one mounted at the end of the fiber bundle (Fig 2, no.2), in order to mimic the incoming plasma light as close as possible in the calibration section. The plasma and calibration light cones experience the same stops in virtue of the lens tandem (Fig, no.3). The remaining discrepancies in the setup between calibration

140 and plasma optics has been proven to not influence the measurements. The proof is based on a comparison of images of the C-Wave laser light through two Ulbricht spheres, the one in the soft iron box (Fig,2, no.1a) and another one placed in the W7-X plasma vessel after the end of the experimental campaign. The measured phase difference during this test remained below $1^{\circ}$, with no variation across the image. The result is therefore equivalent to an apparent velocity below $10 \mathrm{~m} / \mathrm{s}$, which is negligible with respect to the $\mathrm{km} / \mathrm{s}$ plasma flows. A spectral lamp is also present in 145 the systems, in order to perform comparisons between a standard and the new calibration sources.

Calibration measurements of $\lambda_{0}$, performed with the C-Wave laser, are repeated before and after every plasma experiment, in order to have always a reliable reference point even without temperature control devices. The frequent calibration also allows the identification of possible changes in the setup due to systems vibrations (related to e.g. scaffolds movements). The process is controlled with a software developed in LabView, that opens/closes the laser

150 shutter and moves the mirror in the vertical system, while an internally developed software is interfacing the camera and loading the data to the W7-X archive. The calibration images are recorded at fixed time before the plasma starts, $15 \mathrm{~s}$ for the toroidal system and $35 \mathrm{~s}$ for the vertical one, as well as after the plasma ends, $5 \mathrm{~s}$ for the toroidal system and $25 \mathrm{~s}$ for the vertical one.

In the next sections, the toroidal system will be use as main example, as its typical flow pattern has been already 155 explained in [17. Flow measurements performed with the vertical system are expected to be topic of future physics 


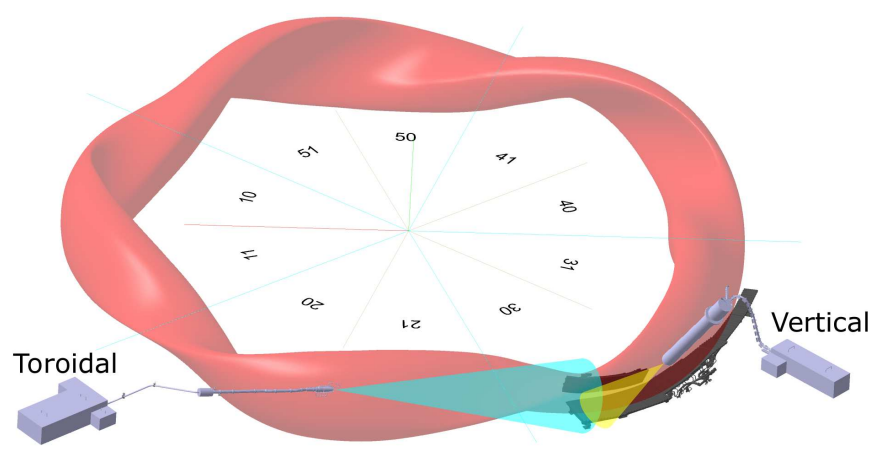

(a)

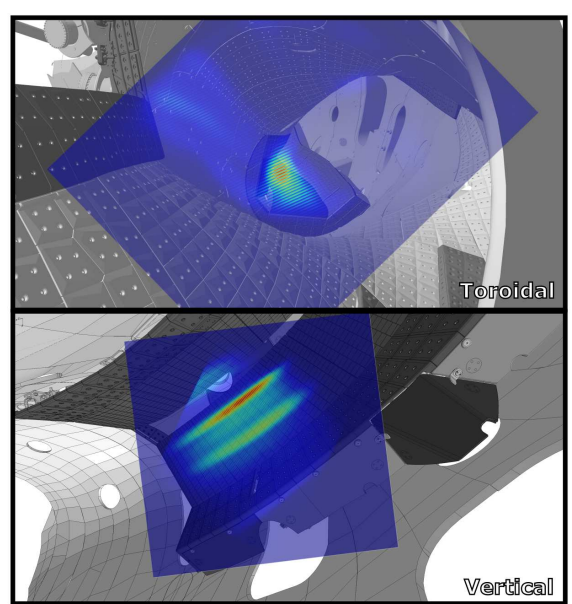

(b)

FIG. 3. Views of the two CIS systems at W7-X. (a) The CIS systems are represented by the soft iron boxes and the immersion tubes, where the image fiber bundles are laid to observe the plasma. The red part represents the core plasma shape (i.e. excluding the SOL). At the center, the labels indicating the names of the machine modules are displayed. The cone of view is represented in cyan and yellow for the toroidal and vertical systems respectively. The black component is the island divertor module under investigation (namely, lower module 3). (b) Raw data of CIS camera images overlaid to the CAD model of the interior of the machine.

papers.

\section{A. Geometry in W7-X}

The two installed systems are viewing the same regions (called 1-2v and 1-2-3h as defined in 28 ) of one of the island divertors of W7-X (namely, lower in module 3). A representation of the viewing chords can be seen in Fig. 3 . 160 As can be seen from the raw data overlaid on the CAD model of the interior of the machine, the toroidal system has a good view on the entire SOL, while the vertical one is focused on a small section of the divertor and of the outer SOL.

The toroidal and vertical systems cover respectively toroidal angles from $\phi=72^{\circ}$ to $\phi=145^{\circ}$ (equivalent to an entire module) and from $\phi=125^{\circ}$ to $\phi=136^{\circ}$ (restricted to a small region of one divertor), where the angle is defined,

165 looking down from above, as increasing counter-clockwise, with $\phi=0^{\circ}$ being set between the W7-X half-modules 10 and 11. This translates in a substantial difference in the length of the lines of sight between the two views: the distance between the diagnostic port of the toroidal system and the divertor is $\sim 4$ times longer. This has a considerable effect on the quality of the contrast of the recorded plasma images. A decrease of $\sim 50 \%$ in contrast with respect to the calibration measurements is in general expected, since this parameter is linked to the spectral line broadening, i.e. the

170 ion temperature in the plasma. However, a non-negligible difference in contrast between the two systems has been highlighted by testing them with the same interferometer cell $(10 \mathrm{~mm}$ thick displacer plate, $6 \mathrm{~mm}$ thick delay plate) during similar plasma experiments. The decrease is evident for the plasma images only, suggesting that the effect goes beyond possible variations in the optical setup of the two CIS boxes. This is shown in Fig 4 (a), where the contrast has been retrieved with a 2D FFT analysis. The plot displays only one column of pixels in the plasma frame, specifically 175 the one passing through the region of the highest brightness for both systems, as shown in Fig 4 (b). As can be seen, the contrast for the calibration images is quite similar (between $60-70 \%$ ), while the contrast for the toroidal view is almost half of the one of the vertical view during plasma (from $\sim 9 \%$ to $\sim 2 \%$ ), resulting in barely visible fringes and therefore low signal-to-noise ratio. This justifies the choice of two different interferometer cells for the two systems: decreasing the amount of crystals improves the contrast, at the cost of a lower velocity sensitivity. For the vertical 180 view, a higher sensitivity (roughly doubled with respect to the one of the toroidal system) is preferable because the observed flow velocity is largely perpendicular to the magnetic field and, therefore, is expected to be lower.

The final setup led to an average contrast of $\sim 40 \%$ (plasma) $\sim 85 \%$ (calibration) for the toroidal system and of $\sim 15 \%$ (plasma) $\sim 65 \%$ (calibration) for the vertical one. The substantial lower contrast during plasma experiment is not fully tackled here and it will be subject of future investigations. 


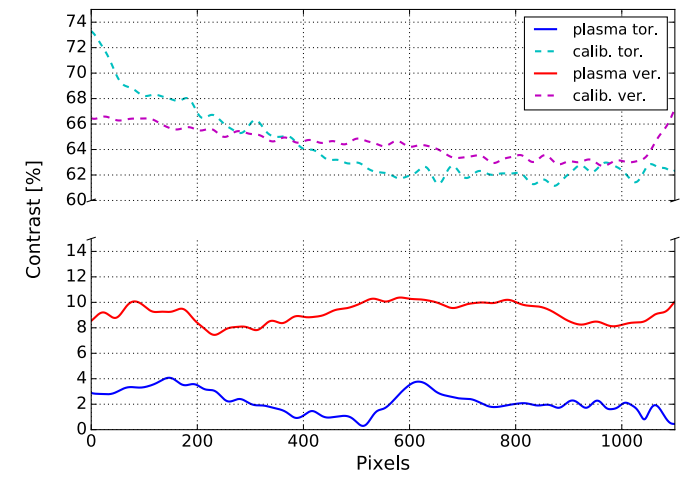

(a)

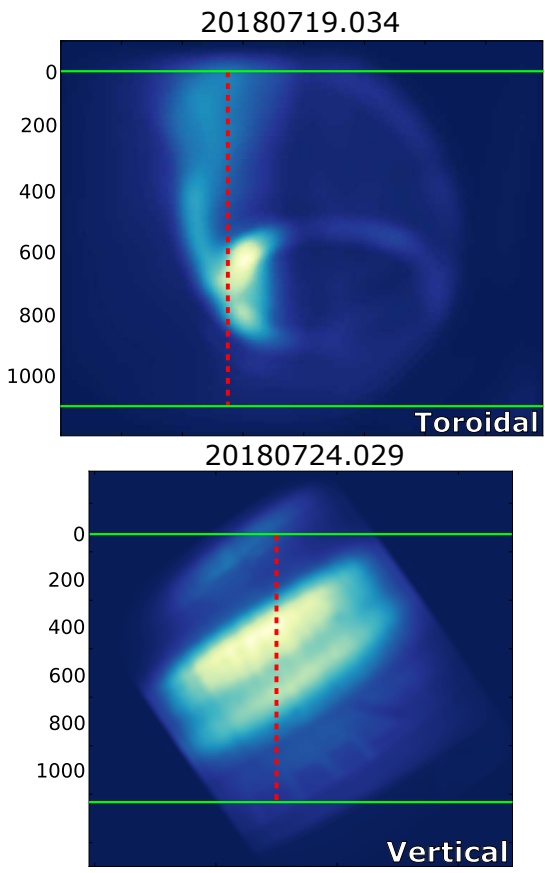

(b)

FIG. 4. Contrast study, with the same interferometer cell (10 mm thick displacer plate, $6 \mathrm{~mm}$ thick delay plate) used in the two systems. The plasma experiments (20180719.034 for the toroidal system and 20180724.029 for the vertical one) had similar parameters (standard magnetic configuration, input power $\sim 2 \mathrm{MW}$, line integrated electron density $\sim 2 \cdot 10^{19} \mathrm{~m}^{-2}$, ion temperature in the plasma core $\sim 1.5 \mathrm{keV}$ ). The C III plasma radiation was measured in both cases. (a) Comparison between the two systems. The contrast is displayed for a calibration image (dashed line) and for a plasma image (solid line) for each system. The study is restricted to a single column of pixels in the frame, highlighted in (b) (red dash line between the two green lines). The pixels have been selected in a region of high brightness. (b) Snapshot of the plasma brightness for both systems.

As previously mentioned, the filter used in CIS needs to exclusively transmit the emission of one chosen charge state of one chosen species, otherwise the measurements might be corrupted. An example of disturbed data can be seen in Fig.5. During this experiment, nitrogen was puffed from the He Beam system 29] placed in the same divertor module imaged by CIS. Nitrogen has emission lines (N III at $463.413 \mathrm{~nm}$ and $464.064 \mathrm{~nm}$ ) that enter the filter of C $190 \mathrm{III}$, centered at $465 \mathrm{~nm}$ and $2 \mathrm{~nm}$ wide. Due to the puff, the nitrogen content was locally high enough to influence the C III flow measurement. The result is a local intensity change in the counter-streaming velocity pattern.

Another example of impurity disturbance for the C III measurements can be seen by comparing the contrast value for experiments before and after boronization. At W7-X, boronization successfully reduced the release of impurity from the walls during the experiments, in particular oxygen [30. Oxygen has emission lines that can enter in the filter 195 of C III (O II at 463.8855, 464.181, 464.3386, 464.9135, 465.0839 nm). The spectrum has been measured by standard high-resolution spectroscopy, which identified an oxygen contribution close to the C III center of mass, characterized by a line intensity at least $\sim 7$ times smaller than the carbon one. The presence of this impurity affects the contrast, i.e. it induces a change in effective measured line broadening. Fig. 6 shows two equivalent plasma discharges, one before and one after the first boronization at W7-X. In the improved machine conditions, an increase of the contrast 200 of $\sim 2-3 \%$ is observed. In the pre-boronization experiments, the presence of oxygen might have altered the phase as well, even though it was not possible to identify any effect on the flow measurements. Therefore, the results from these experiments should be handled with care and are not considered for quantitative analysis in the rest of the paper. 


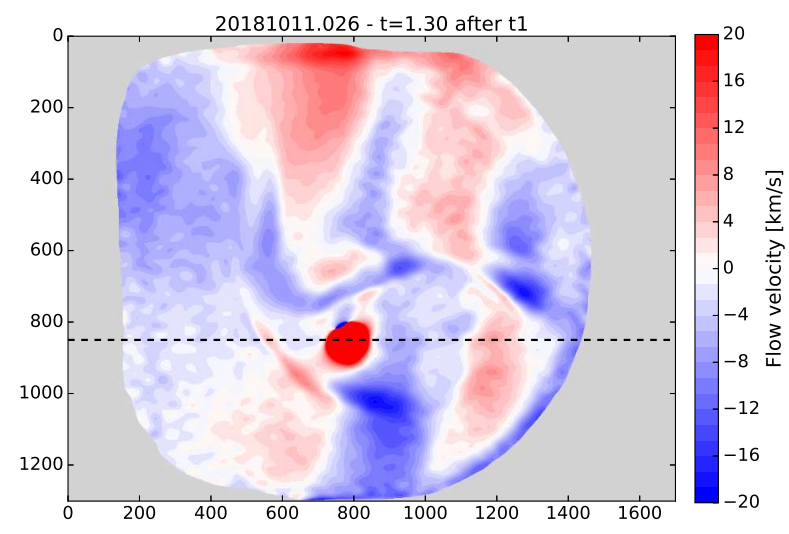

FIG. 5. Evidence of impurity corruption in a CIS flow measurement. Single camera frame of experiment 20181011.026 in the case of the toroidal system, during which nitrogen was puffed from the He Beam system in the lower divertor of module 3 . The colorbar limits are set in order to display the counter-streaming flows. The location of the nitrogen puff (centered in (800, 850) pixels) gives unphysical values of up to $70 \mathrm{~km} / \mathrm{s}$.

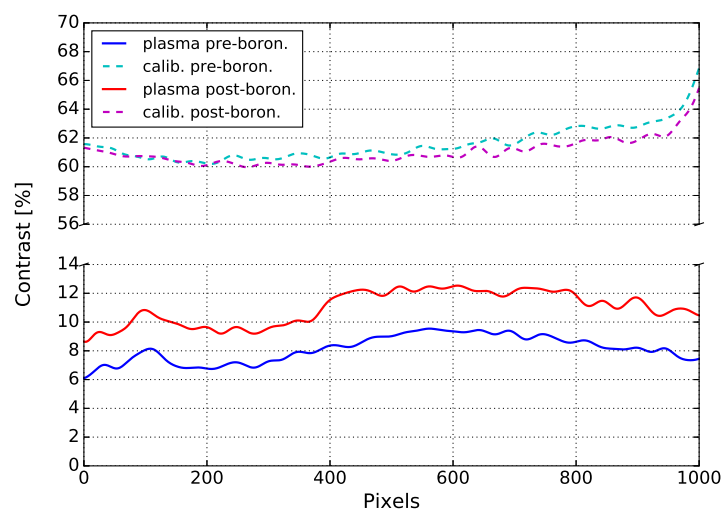

FIG. 6. Contrast variation due to boronization. The contrast study, done for the vertical system, is restrict to a single column of pixels in the plasma frame, passing through the region of highest intensity. The plasma experiments (20180801.021 as preand 20180807.007 as post-boronization) had similar parameters (standard magnetic configuration, input power $\sim 2 \mathrm{MW}$, line integrated electron density $\sim 2 \cdot 10^{19} \mathrm{~m}^{-2}$, ion temperature in the plasma core $\sim 1.5 \mathrm{keV}$ ). The contrast is displayed for a calibration image and for a plasma image for both discharges.

\section{LASER CALIBRATION MEASUREMENTS}

205 In order to obtain the flow velocity values from the measured data, a reliable calibration process is required. The CIS system is inducing a phase $\Phi_{\mathrm{p}}$ between the two polarization states of the incoming plasma light, that depends on its wavelength $\lambda_{\mathrm{p}}$. The Doppler velocities are defined as $v_{\text {ion }}=c \cdot\left(\lambda_{\mathrm{p}}-\lambda_{0}\right) / \lambda_{0}$, where $\lambda_{0}$ is the unshifted wavelength emitted by the ion at rest. Therefore, it is necessary to know the phase $\Phi_{0}$ induced by the birefringent plates for $\lambda_{0}$. The relation $\Delta \Phi \rightarrow \Delta \lambda$ depends on parameters that are not normally known with high enough precision [26]. The 210 most reliable approach is hence to have a calibration source with emission lines as close as possible to $\lambda_{0}$.

The C-Wave laser used at W7-X is an alternative approach to reach the level of precision needed for a reliable calibration process for many different $\lambda_{0}$. The C-Wave stability has been proven by monitoring its emission during 8 hours of continuous operation: according to the wavemeter, the wavelength variation remained below $4 \mathrm{fm}$, a quantity that is not resolvable with the W7-X CIS systems. Any mechanical movement (e.g. the shutter) can momentarily affect

215 the emission stability, but the desired wavelength is reached again in few seconds by virtue of the AbsoluteLambda software. Thanks to this high accuracy, the laser can be used as a reference also to investigate how the temperature affects the CIS function.

The C-Wave laser can also perform a direct measurement of wavelength shifts by fine scanning in the same range as the Doppler drifts expected by impurity ions in plasma discharges ( $\pm 30 \mathrm{pm}$ in $5 \mathrm{pm}$ steps). This can highlight how 
220 the CIS systems are responding to different wavelengths and it is critical in accurately calibrating both the direction and the zero point of the impurity flow velocity [16.

A collection of measurements performed with the two CIS systems and the C-Wave are presented in the next subsections.

\section{A. Comparison with standard calibration method}

225 Previous CIS applications used standard spectroscopy lamps or single emission lasers as calibration sources [9, 24]. In particular, for the measurement of the C III line, zinc lamps were preferred, having an emission line at $468.0136 \mathrm{~nm}$, $\sim 3 \mathrm{~nm}$ away from the C III center of mass at $464.8811 \mathrm{~nm}$ [13. Although the C-Wave laser makes such a Zn lamp calibration obsolete, it is illuminating to use the laser to examine and improve the $\mathrm{Zn}$ lamp technique for uses where the $\mathrm{C}$-Wave laser is not available.

The use of calibration sources for flow velocity measurements with even a few nm difference, such as the Zn lamp for calibrating the C III spectra line, has been shown to require complex corrections that cannot always be determined precisely [13, 31].

The corrections are related to an offset in the phase induced by the birefringent plates. The quantity that needs to 235 be known to obtain the Doppler velocities is

$$
\Delta \Phi_{\mathrm{D}}=\Phi_{\mathrm{p}}-\Phi_{0}
$$

while the quantity measured with a spectral lamp calibration source is

$$
\Delta \Phi_{\mathrm{c}}=\Phi_{\mathrm{p}}-\Phi_{\mathrm{Zn}}
$$

where $\Phi_{\mathrm{Zn}}$ is the phase induced by the birefringent plates for the Zn lamp spectral line. Therefore, it is necessary to know the quantity

$$
\Delta \Phi_{\mathrm{Zn}}=\Phi_{\mathrm{Zn}}-\Phi_{0}
$$

to obtain $\Delta \Phi_{\mathrm{D}}$, that will be

$$
\begin{aligned}
\Delta \Phi_{\mathrm{D}} & =\Delta \Phi_{\mathrm{C}}+\Delta \Phi_{\mathrm{Zn}} \\
& =\Phi_{\mathrm{p}}-\Phi_{\mathrm{Zn}}+\Phi_{\mathrm{Zn}}-\Phi_{0} .
\end{aligned}
$$

240 Without the C-Wave laser, $\Delta \Phi_{\mathrm{Zn}}$ is normally simulated [13, 31]. This simulated quantity depends on the plate parameters which are not known with sufficient precision. The parameters are instead fit to one or more calibration sources and it has been shown that the fit is unique and sufficient to correctly reproduce $\Delta \Phi_{\mathrm{Zn}}$ [31. Unfortunately, these procedures are complicated and time consuming, as the simulation is highly sensitive to the plate parameters (especially when $\lambda_{\mathrm{Zn}}-\lambda_{0} \geq 1 \mathrm{~nm}$ ) and it needs to be repeated for every plasma wavelength under investigation [31.

245 Here, $\Delta \Phi_{\mathrm{Zn}}$ has been measured directly for the first time. Since the laser can be tuned exactly to the center of mass of C III, the measurement was possible by fast switching between the $\mathrm{Zn}$ lamp and C-Wave laser emissions in both CIS systems. The result can be seen in Fig.7 The $\sim 3 \mathrm{~nm}$ difference in wavelength distorts the measurement up to the point that $\Delta \Phi_{\mathrm{Zn}}$ is so steep that a phase jump must exist within the image. The discrepancies between the two systems are related to the different optical configurations, which also lead to different equivalent velocities $250\left(\Delta \Phi=180^{\circ}\right.$ is equivalent to $80 \mathrm{~km} / \mathrm{s}$ for the toroidal system and to $40 \mathrm{~km} / \mathrm{s}$ for the vertical one). The phase jump appears to be aligned to the fringe direction in the raw data.

The same type of phase jump appears if a plasma frame is calibrated with the Zn lamp without any correction applied (i.e. in $\Delta \Phi_{\mathrm{C}}$ ), as shown in Fig 8 (b). Once $\Delta \Phi_{\mathrm{Zn}}$ is applied, like in equation (4), the expected counter-streaming flow pattern [17] becomes once more visible, as shown in Fig.8(c). This implies that the effect introduced by the lamp

255 is possible to correct despite the temperature dependence of the phase. If the dependencies are made explicit, with $T_{0}$ and $T_{1}$ defined as temperature values in different moments in time (e.g. during different experimental days), equation (4) becomes

$$
\begin{aligned}
\Delta \Phi_{\mathrm{D}} & =\Delta \Phi_{\mathrm{C}}\left(T_{1}\right)+\Delta \Phi_{\mathrm{Zn}}\left(T_{0}\right) \\
& =\Phi_{\mathrm{p}}\left(T_{1}\right)-\Phi_{\mathrm{Zn}}\left(T_{1}\right)+\Phi_{\mathrm{Zn}}\left(T_{0}\right)-\Phi_{0}\left(T_{0}\right)
\end{aligned}
$$




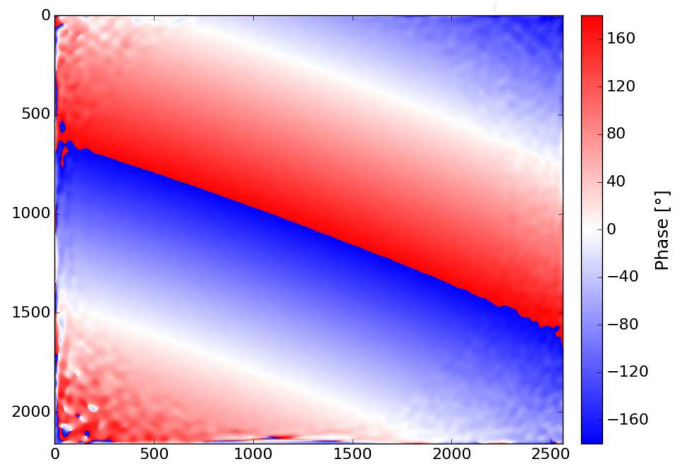

(a)

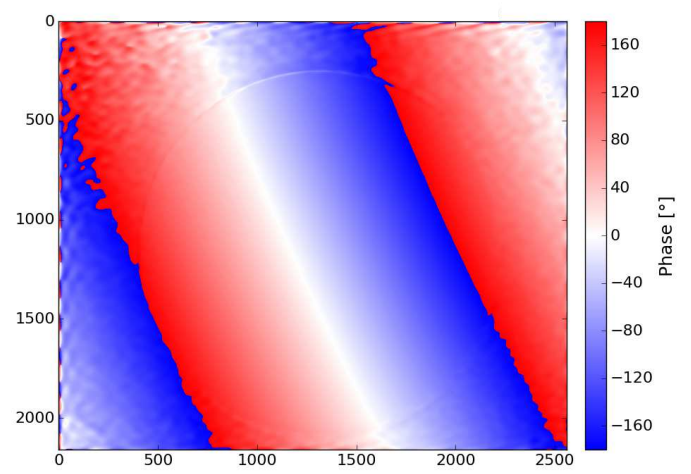

(b)

FIG. 7. Measured phase difference $\Delta \Phi_{Z n}$ between the Zn lamp line at $468.0136 \mathrm{~nm}$ and the C III multiplet center of mass at $464.8811 \mathrm{~nm}$. (a) For the toroidal system; (b) for the vertical system.

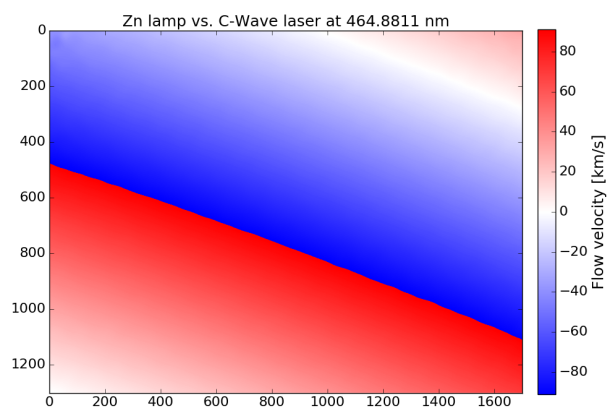

(a)

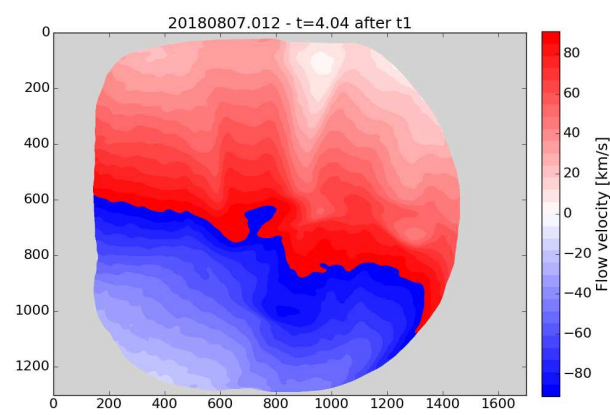

(b)

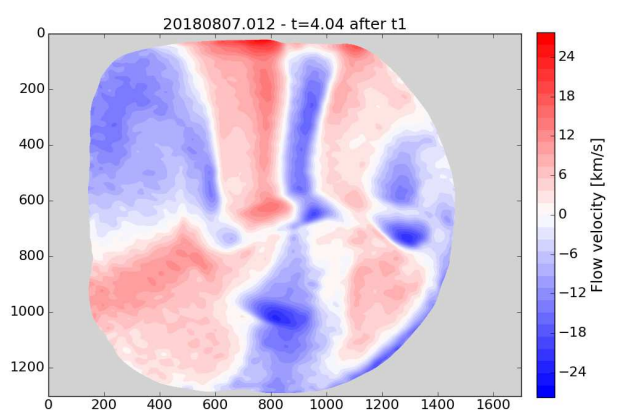

(c)

FIG. 8. Applied $\Delta \Phi_{Z n}$ on the toroidal system. (a) $\Delta \Phi_{Z n}$ translated in velocities and zoomed in the camera chip region covered by the image guide. Example of plasma measurement calibrated with a Zn lamp (b) without and (c) with the measured correction.

where $\Phi_{\mathrm{p}}\left(T_{1}\right)-\Phi_{0}\left(T_{0}\right)$ is not the desired quantity $\Delta \Phi_{\mathrm{D}}$ and the terms $\Phi_{\mathrm{Zn}}\left(T_{1}\right)$ and $\Phi_{\mathrm{Zn}}\left(T_{0}\right)$ do not cancel out. However, the result shown in Fig, demonstrates that $\Delta \Phi_{\mathrm{Zn}}$ is equal at any different temperature, and any effect 260 induced by $T_{1} \neq T_{0}$ is taken into account through the quantity $\Phi_{\mathrm{Zn}}\left(T_{0}\right)-\Phi_{\mathrm{Zn}}\left(T_{1}\right)$. This has been confirmed by applying equation (4) to other plasma discharges, always obtaining the counter-streaming flow pattern as a result.

The term $\Delta \Phi_{Z n}$ has been measured also for the spectral line He II at $468.57 \mathrm{~nm}$. Being only $\sim 0.5 \mathrm{~nm}$ away from the multiplet center of mass, the effect is less prominent but still non-negligible. In this case no phase jump is present, but the phase still varies from $-100^{\circ}$ to $-50^{\circ}$ across the camera chip, again following the fringe pattern direction.

\section{Effect of the new laser calibration on fringe contrast}

Up to this point, the discussion considered the contrast only as how distinguishable the fringes of the interference pattern are. Nonetheless, this parameter is related to the broadening of the investigated spectral line and can therefore give information on the ion temperature [22. For this reason, having a good calibration will help in implementing a CIS system able to measure ion temperatures.

270 At W7-X, the CIS systems have been optimized to measure ion flow velocities, thus they are less sensitive to contrast variations than to phase changes. However, it has been possible to observe an improvement of the contrast quality when the C-Wave laser is used instead of a spectral lamp. The C-Wave narrow emission affects positively the contrast when compared to the Zn lamp measurements: the contrast increases of $\sim 3 \%$ for both systems, leading to nearly $90 \%$ and $70 \%$ for the toroidal and vertical system respectively. The noise level is also reduced, confirming once more the 275 value of the new W7-X calibration source. 


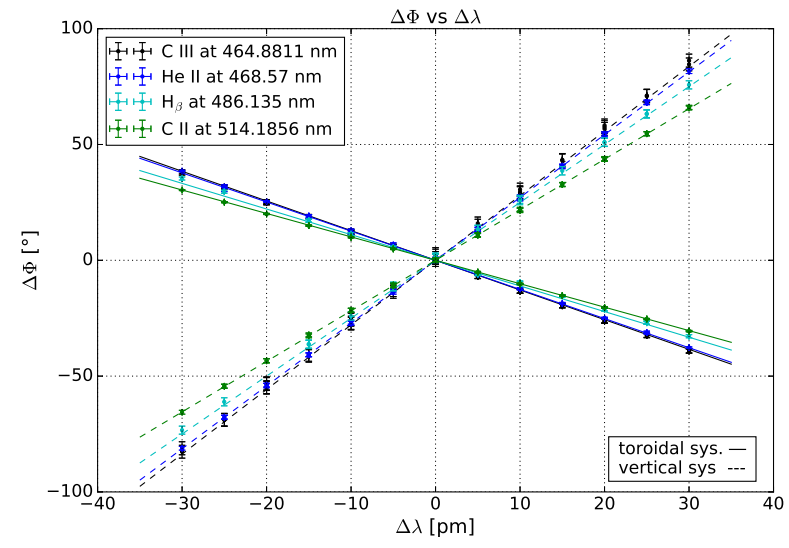

FIG. 9. Wavelength scans performed with the C-Wave laser for toroidal and vertical systems.

\section{B. Wavelength scans}

Small-range wavelength scans are useful to characterize the CIS phase response and consequently be able to translate phase variations into flow velocity without relying on simulations. This type of dispersion measurement has been already presented in [16] for one line of interest, and it is here repeated for different central wavelength in order to 280 highlight a wavelength dependence of the CIS response. The C-Wave laser has been tuned $\pm 30 \mathrm{pm}$ around $464.8811 \mathrm{~nm}$

(C III), $468.57 \mathrm{~nm}$ (He II), $486.135 \mathrm{~nm}\left(\mathrm{H}_{\beta}\right)$ and $514.1856 \mathrm{~nm}$ (C II) in $5 \mathrm{pm}$ steps. An additional fixed laser source (HeNe with emission at $633 \mathrm{~nm}$ ) has been used as a reference to monitor potential phase variations due to ambient temperature changes. The measurement lasted 10 minutes for every central wavelength. The results are displayed in Fig 9, where the error bars represent the statistical error and take into account phase variations of maximum $0.8^{\circ}$ 285 and $2.5^{\circ}$ for the toroidal and vertical systems respectively, which are induced by the boxes temperature change. The opposite slopes are related to the different positions of the crystals optical axis in the two systems. Fig 9 shows that the velocity sensitivity decreases for higher wavelength: a variation of $\sim 50 \mathrm{~nm}$ from blue to green wavelengths corresponds to a reduction of $\sim 20-22 \%$ in velocity sensitivity. This means that, in order to have a precise translation from phase to velocity measurements, wavelength scans need to be repeated for every plasma spectral line under investigation. 290 This is easily doable with the C-Wave laser, however not with spectral lamps or diode laser applications.

\section{Temperature effects}

CIS has been shown to be very sensitive to temperature variations [24]. The refractive indices $n_{\mathrm{e}}$ and $n_{\mathrm{O}}$ of the birefringent crystals change significantly with temperature even for few degrees centigrade. Attempts of minimization for these effects have shown that they cannot be entirely suppressed [15. Instead, at W7-X, no temperature control 295 devices are installed and the chosen solution is to perform frequent calibration measurements, before and after every plasma experiment. The reliability of this method is checked by comparing the images recorded during the two calibration sessions. As the longest plasma of OP1.2 was $100 \mathrm{~s}$ long [32, no perceptible changes were observed on these timescales. Nonetheless, temperature effects might play a major role in the future, since W7-X has been designed to perform 30 minutes long plasma discharges. This led to further investigations on the effect of temperature variations 300 throughout an experimental day. As previously stated, the W7-X CIS systems have been optimized for velocity flow measurements, therefore the studies presented here are centered on the phase parameter. The contrast behavior, which showed similar but less prominent tendencies, will be topic of future work.

The phase variation has been analysed for different experimental days. The temperature effect can be highlighted by comparing the calibration data to the first calibration image of the day. An example of these studies is shown in 305 Fig 10, in which phase variations are compared, for both systems, with the laser output wavelength and surrounding ambient temperature recorded inside the soft iron box every 5 minutes. The laser, set at $464.8811 \mathrm{~nm}$, is confirmed to be stable throughout the experimental day, with variations below $10 \mathrm{fm}$ even considering the mechanical disturbance of the shutter. The relative changes in phase are therefore only linked to the ambient temperature fluctuations. The effect scales with the number of crystals used: for a $1^{\circ} \mathrm{C}$ difference, the phase varies of $\sim 40^{\circ}$ for the toroidal system and 310 of $\sim 80^{\circ}$ for the vertical one, that translates in both cases to $\sim 18 \mathrm{~km} / \mathrm{s}$. The sign of the variations (negative/positive for the toroidal/vertical systems) fits the sign of the slopes found in the wavelength scans (see Sec III B. 


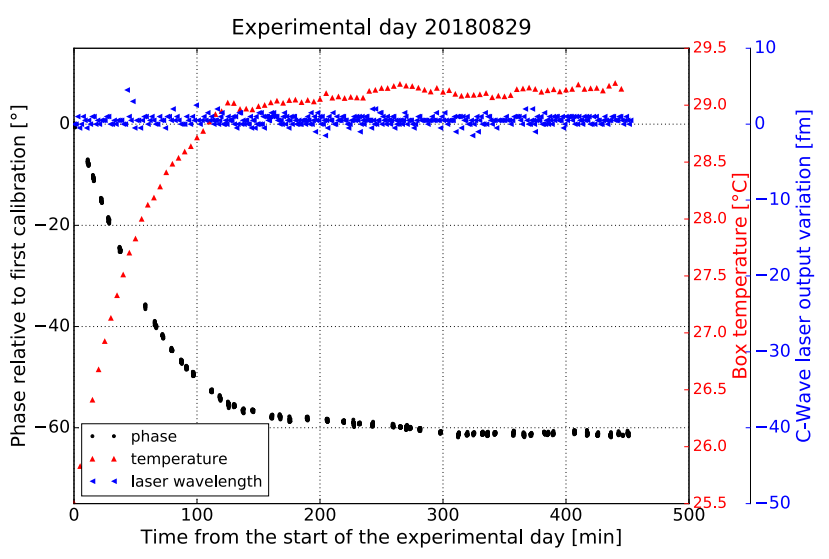

(a)

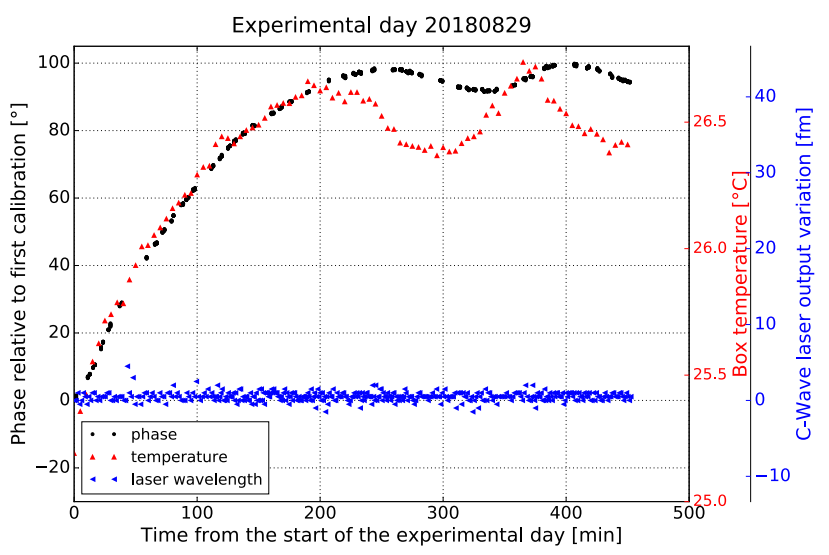

(b)

FIG. 10. Phase variation during an experimental day due to temperature changes in the soft iron box. The phase is evaluated in an area of 20x20 pixels in the center of the images. Analysis of calibration images only, quantity displayed relative to the first calibration image of the day. The laser trace is plotted with one point every 2000. (a) For the toroidal system; (b) for the vertical system.

In order to highlight the correlation of CIS phase and ambient temperature, dedicated experiments with the vertical system have been carried out with a temperature sensor characterized by a higher sampling rate (i.e. able to record every $15 \mathrm{~s}$ ), placed close to the interferometer cell. The measurements have been performed with two wavelengths at 315 same the time, with the purpose of investigating if the effect is wavelength dependent. This is possible by using two lasers: the C-Wave and a HeNe one (with emission at $632.816 \mathrm{~nm}$ ). If the $\mathrm{C}$-Wave is set in the blue/green range, the wavelengths of the two lasers are well distinguishable in the 2D FFT spectrum [16. The chosen wavelengths for the C-Wave are $464.8811 \mathrm{~nm}$ (equivalent to the C III center of mass) and $514.1856 \mathrm{~nm}$ (equivalent to the C II center of mass). The data for the two wavelengths has been collected in two different days. The temperature variation has not

320 been controlled, so the recorded increase is only due to the diagnostic electronics, running for 6 hours. The results are shown in Fig 11, where the data are displayed together with dedicated simulations based on the equation (12) in [26], describing the phase induced by birefringent crystals. The temperature dependence has been taken into account in the calculation of the refractive indices of the $\alpha \mathrm{BBO}$ crystals and of air. The points are displayed relatively to the first image of the data set. It can be seen that CIS is sensitive to small temperature variations, as a non-negligible

325 phase difference on the order of $10^{\circ}$ is induced even for a $0.2^{\circ} \mathrm{C}$ change. The effect appears to be reproducible, as the trace of the HeNe laser shows the same evolution for both experiments (e.g. $0.4^{\circ} \mathrm{C}$ induces a phase difference of $40^{\circ}$ in both cases). Despite a clear wavelength dependence $\left(0.5^{\circ} \mathrm{C}\right.$ induces $\sim 24^{\circ}, 29^{\circ}, 32^{\circ}$ respectively for red, green, blue wavelengths), the temperature affects the velocity measurement always in the same way: the less strong variation measured for wavelengths towards the green/red range is compensated by the decreased sensitivity to wavelength

330 displacements shown in Sec III B. This translated in having $6 \mathrm{~km} / \mathrm{s}$ for $0.4^{\circ} \mathrm{C}$ in all the cases. The theoretical curves reproduce the effect, but an overestimation is stronger for smaller temperature changes, probably due to the fact that other parameters (e.g. the plate thickness, the stress on the crystals due to mechanical mountings, ...) could be influential as well.

The reproducibility of the temperature effects on the phase behavior and the good theoretical representation 335 achieved here give the basis to develop a reliable method to face the challenge of the 30 minutes operation foreseen for W7-X without expensive temperature control techniques.

\section{Multiplet measurements}

As first reported in 22, measuring spectral lines that are not singlet can introduce an offset depending on the CIS crystal configuration. This is due to the assumption that the interference pattern generated by the multiplet center 340 of mass $\left(I_{0}\right)$ is equivalent to the superposition of interference patterns generated by all the multiplet components $\left(I_{\mathrm{M}}\right)$, which is not always holding. This effect can be simulated [13, 31] and the correction is reliable if the crystal parameters are well-known.

Knowing the crystals parameters from a fit of the wavelength scans presented in Sec IIIB, it is possible to calculate the phase $\Phi\left(I_{\mathrm{M}}\right)=\Phi_{\mathrm{M}}$ induced by the multiplet structure of the plasma spectral line. The offset phase $\Delta \Phi_{\mathrm{M}}$ is 


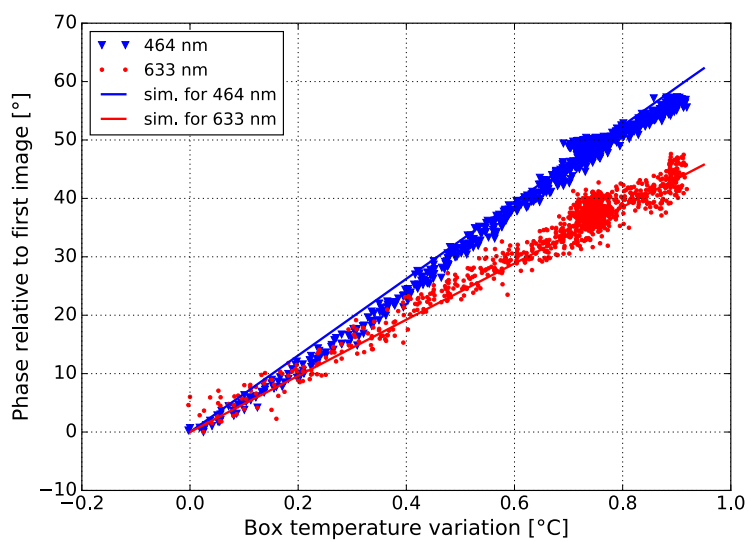

(a)

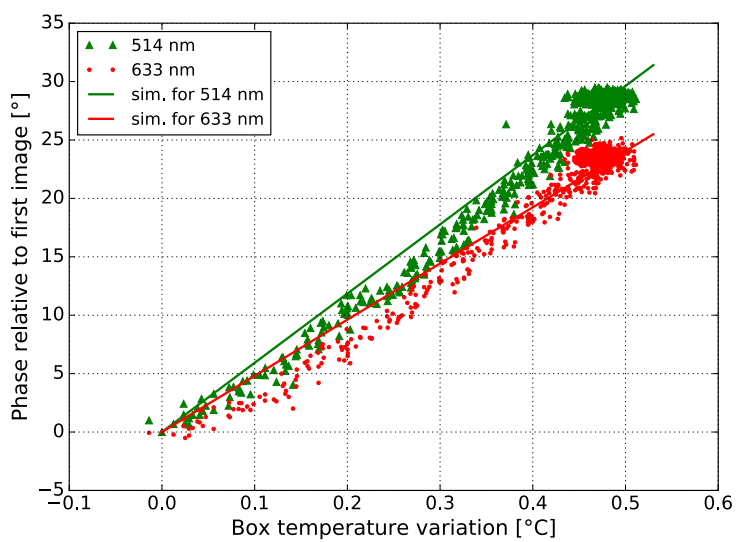

(b)

FIG. 11. Phase variations with ambient temperature changes. The phase is evaluated in an area of $20 \mathrm{x} 20$ pixels of the images. Measurement performed with the vertical system. Two wavelengths are measured at the same time, with the use of the C-Wave and a HeNe lasers. C-Wave set at (a) $464.8811 \mathrm{~nm}$; (b) $514.1856 \mathrm{~nm}$.

345 defined as

$$
\Delta \Phi_{\mathrm{M}}=\Phi_{\mathrm{M}}-\Phi_{0}
$$

where $\Phi_{0}=\Phi\left(I_{0}\right)$, as defined in Sec.III A 1. Therefore, the desired quantity $\Delta \Phi_{\mathrm{D}}$ needs to be corrected in the following way:

$$
\Delta \Phi_{\mathrm{D}}=\Phi_{\mathrm{p}}-\Phi_{0}-\Delta \Phi_{\mathrm{M}}
$$

The correction factor $\Delta \Phi_{\mathrm{M}}$ can be applied manually during the data analysis process. Another approach would be calibrating by reproducing the multiplet, one component at a time. This is prevented by the fact that the multiplet 350 components often differ of more $200 \mathrm{pm}$, which is a range that the C-Wave tuning process does not cover in a time interval short enough to avoid temperature drifts. At W7-X, the laser was set to a shifted wavelength that induces the same phase as $\Delta \Phi_{\mathrm{M}}$. The result translates in having

$$
\Delta \Phi_{\mathrm{D}}=\Phi_{\mathrm{p}}-\Phi\left(\lambda_{\mathrm{eff}}\right)
$$

where $\Phi\left(\lambda_{\text {eff }}\right)=\Phi_{0}+\Delta \Phi_{\mathrm{M}}$.

The most illustrative example is the multiplet effect for the C III emission. The three spectral lines generated by 355 the same atomic transition are characterized by a highly asymmetric structure. This can cause a non-negligible offset depending on the crystal setup. For example, for the case of the W7-X toroidal system, $\Delta \Phi_{\mathrm{M}}=-170^{\circ}$. Relying on the wavelength scans, a phase of $-170^{\circ}$ is equivalent to a $\Delta \lambda \approx 130 \mathrm{pm}$. Therefore, $\lambda_{\text {eff }}$ can be set to $464.75 \mathrm{~nm}$ to compensate the multiplet effect.

The use of the $\lambda_{\text {eff }}$ approach allows to ease the data analysis, as the simulation of $\Delta \Phi_{\mathrm{M}}$ is needed only once. On 360 the other hand, this method still depends on how precisely the plate parameters are known.

\section{E. Determination of flow direction}

The direct measurement of the relation between $\Delta \Phi$ and $\Delta \lambda$ shown in Sec IIIB allows to identify the correct sign of the measured Doppler displacements without ambiguity. Once the sign is correct, it is possible to determine the direction of the impurity flows with respect to the in-vessel components or the magnetic field by performing a 365 geometrical analysis. The example of the toroidal view is reported here, in the specific case of carbon impurity in a SOL characterized by 5 independent magnetic islands (standard magnetic configuration [33]).

The CIS measurements are line-of-sight integrated. The integration over multiple regions of different flows and temperatures is translated in a complex superposition of different spectral components. The effective inferred single velocity value is in general not simply the line averaged velocity, as typically assumed in simulations [17. Consequently, 370 some kind of localization is necessary for carrying the geometrical analysis. On the other hand, the CIS measurements 
(a)

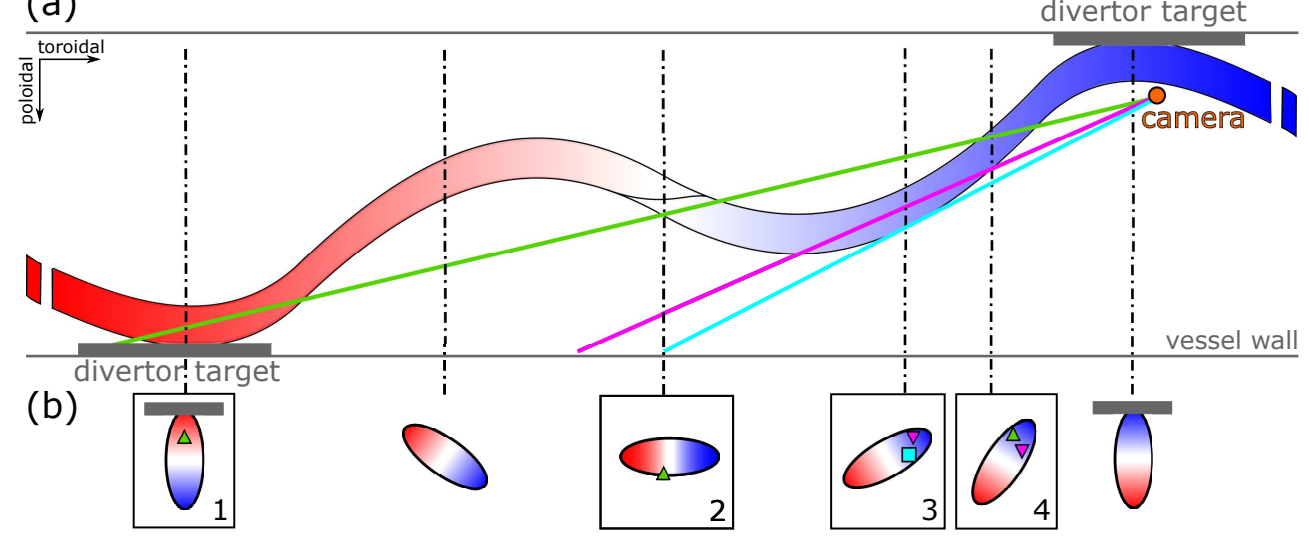

FIG. 12. Sketch illustrating the relation between CIS lines of sight and magnetic islands for the toroidal system. The orange dot indicates the camera position. The grey lines serve as the vessel (thinner) and the divertor targets (thicker). The blue and red colors represent the two opposite flow directions. (a) Simplified view from the top of a portion of an island winding around the machine, connecting two divertor targets. 3 different types of lines of sight are shown in green, magenta and cyan. (b) Representation of the island poloidal rotation, with markers indicating the intersection between the island and the lines of sight. The poloidal sections show how each island has both flow directions, as it is cut by different divertor targets in different regions. The green line of sight (triangle with top vertex) intersects the island in sections 1,2 and 4 , crossing regions with opposite flow directions. The magenta line (triangle with bottom vertex) crosses the island in sections 3 and 4 , i.e. it measures only one flow direction but it averages on many poloidal cross-sections. The cyan line (square) intersects the island only in section 3, representing the ideal type of lines of sight for the geometrical analysis.

depend on where the carbon radiates. The C III photo-emission coefficient peaks for electron temperatures of 10-20 $\mathrm{eV}$, values typically found in the SOL of W7-X 34, 35. Moreover, the magnetic islands are characterized, for standard plasma conditions, by strong pressure gradients along the field lines that enhance the parallel particles flows [17, 36]. Accordingly, flows in the magnetic islands are expected to be the major contributors to the measurements. In order to 375 understand the flows direction, it is therefore necessary to identify a region where the viewing chords are intersecting only one magnetic island before hitting the vessel wall (as the magenta and the cyan lines in Fig 12 ). For some specific lines of sight, the intersection happens at a single poloidal plane (as the cyan line only in Fig.12), which can be used as a starting point for characterizing the island open field lines. The distance from the poloidal plane to the closest divertor target (evaluated along the open field lines) dominates the trajectory of the plasma particles and is thus the 380 quantity that can be related to the flow direction measured by CIS.

Fig. 13 shows the described evaluation. The camera looks towards the divertor in the counter-clockwise direction, which coincides with the standard magnetic field orientation. The open field lines taken into consideration are determined by generating scattered starting points around the line of sight intersection with the magnetic island. It can be seen that CIS measures particles streaming towards the divertor targets, since when the closest module is 385 reached going along the magnetic field direction, CIS shows a flow directed away from the camera and vice-versa. It is also possible to notice that the interpretation of the CIS measurement is in general non-trivial, as two points relatively close in the flow image represent two lines of sight that cross the islands in different parts of the machine. Despite the first appearance, the measured image does not represent a poloidal cross-section.

The same analysis has been repeated for different lines of sight and W7-X CIS systems: the carbon flow direction 390 towards the divertor target has been confirmed for standard plasma conditions.

\section{DIAGNOSTIC COMPARISON}

The CIS measurements at W7-X have previously been compared to dedicated EMC3-EIRENE simulations, showing good agreement [17. Additional comparisons with other flow diagnostics can further confirm the quality of the CIS measurements [25]. Suitable candidates are the Mach probes, mounted on the Multi-Purpose Manipulator (MPM) of 395 W7-X [34, 37, 38. Good agreement between CIS and Mach probes has already been observed in a recent investigation on the role of plasma currents for the SOL dynamics in W7-X, where similar changes of the plasma flow caused by increasing plasma currents has been found 39. An additional study is here presented in an attempt to tackle all the aspects that influence the comparison, with a different actuator for changes in plasma flows.

Even though the two diagnostics are both measuring flows, there are substantial differences that prevent a direct 


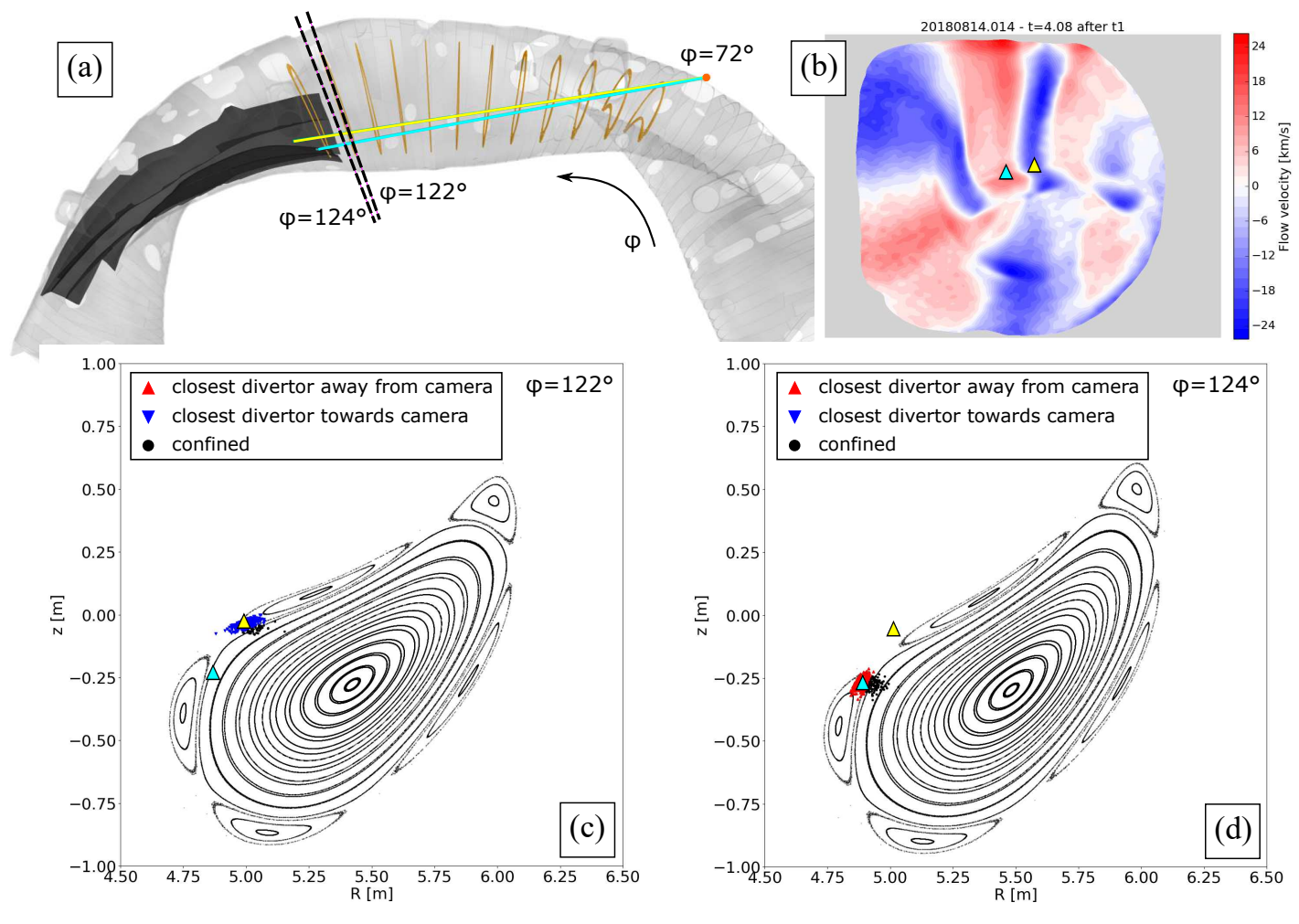

FIG. 13. Geometrical analysis of CIS lines of sight for the toroidal system. For all the plots, the colors red/blue indicate directions inside/outside the paper plane. (a) Plasma vessel of two W7-X modules with representation of two CIS lines of sight in cyan and yellow (view from the top). Poincaré plots of the SOL are displayed in orange between the CIS camera position $\left(\phi=72^{\circ}\right)$ and the beginning of the divertor under investigation $\left(\phi=125^{\circ}\right.$, structure in black). (b) Typical CIS measurement in the standard magnetic configuration. (c), (d) Poincaré plots where the CIS lines of sight intersect one magnetic island. The red/blue markers indicate the direction of the closest divertor target measured along magnetic field lines from the poloidal cross-section. The cyan/yellow markers in (b), (c) and (d) indicate the position of the lines of sight plotted in (a). Note that, in (c), the cyan line of sight does not collect C III emission, as it passes through a too hot plasma region, typically populated by higher ionization stages of carbon. Therefore, it does not contribute to the measured flow image. Similarly for (d), the yellow line of sight lies in a too cold region to contribute to the counter-streaming flow pattern.

400 comparison of the observed absolute velocity values. First of all, the probes measure local radial profiles inside the SOL of W7-X, while CIS displays the line-of-sight integrated behavior of regions of substantial line emission, distributed on the entire edge, which is not a representation of a poloidal cross-section. Moreover, the Mach probes measure the flows of the entire plasma as single ion fluid, relying on the assumption of an isothermal pure hydrogen plasma (which can translate into an ambiguity in the velocity magnitude up to $20 \%$ in typical W7-X experimental conditions), 405 while CIS can investigate the behavior of only one ion species at the time. Furthermore, both diagnostics can show uncertainties in the 0-flow position: the Mach probe flow results can have an offset due to imperfect alignment with the magnetic field, while CIS can present a shift due to the multiplet effect correction. Therefore, the absolute flow velocity values are not expected to agree. In order to be able to correlate the two systems, it is necessary to perform additional geometrical analysis and to find plasmas discharges with a recognisable general tendency of plasma flow 410 variations.

The geometrical analysis is shown in Fig.14. In the standard magnetic configuration, the island crossed by the MPM is connected to the divertor viewed by CIS. In order to monitor the parallel flow in the same island, the measurement performed with the toroidal system needs to be considered, as the vertical one measures perpendicular to the magnetic field. The selected CIS line of sight crosses only the one island, which is required in order to avoid 415 averaging on different flow directions. Unfortunately, the line of sight crosses the island in multiple cross-sections (from $\phi=97^{\circ}$ to $\phi=120^{\circ}$, as highlighted in Fig $14(\mathrm{a})$ ), an additional factor that prevents a direct comparison of absolute velocity values.

The selected plasma discharges are in the standard magnetic configuration, with similar parameters (line integrated density of $7 \cdot 10^{19} \mathrm{~m}^{-2}$, input power of $4 \mathrm{MW}$ of ECRH, electron temperature in the core of $\sim 3 \mathrm{keV}$ ), without (exper420 iment \#20181010.17) and with (experiment \#20181010.18) control coils, which are components designed to induce 


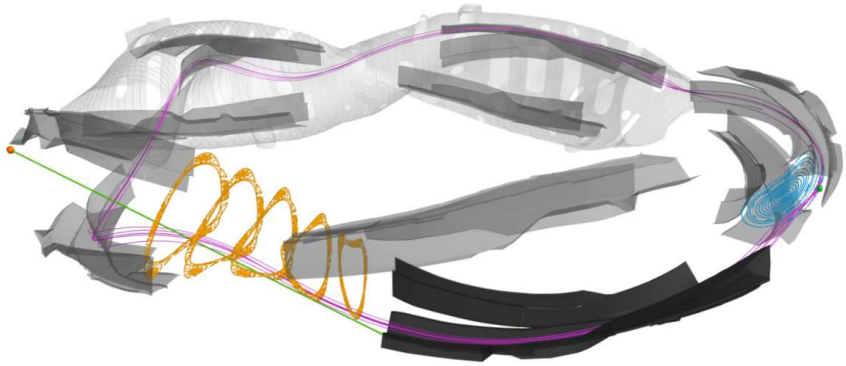

(a)

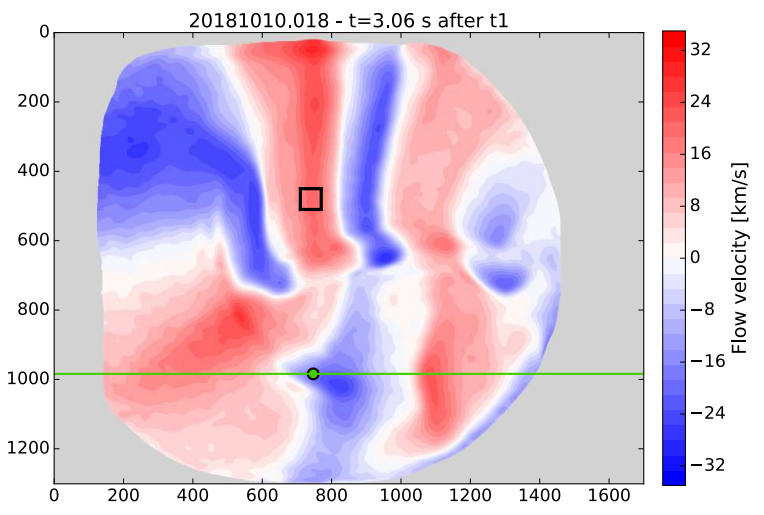

(b)

FIG. 14. Geometrical analysis: identification of a CIS line of sight suitable for comparison with the MPM Mach probes. (a) Schematic of the ten divertor modules with part of the plasma vessel of W7-X (view from the side). The divertor monitored by CIS is highlighted in black. The magnetic island connecting part of the CIS view (green line of sight) to the MPM position (blue Poincaré plot at $\phi=200.8^{\circ}$ ) is traced with magenta lines. Poincaré plots of the SOL are displayed in orange, covering the toroidal angles from $97^{\circ}$ to $120^{\circ}$, where the CIS line of sight crosses the island. (b) CIS flow measurement (single frame) of one of the two analyzed discharges with markers for the investigated line of sight (in green) and for a region of interest that will be used in the discussion (in black).

changes of the island structure. In the latter program, a positive current of $2 \mathrm{kA}$ has been applied to all 10 control coils which results in an increase of the radial component of the magnetic field, increasing the island size while decreasing the connection lengths to the divertors [33, 40. The modification of the SOL geometry translates in an effect on the flows, without modifying the number of islands crossed by the CIS lines of sight.

425 A C III filter was used for CIS in these experiments, therefore the diagnostic monitored the behavior of the carbon impurity. As shown in 41, 3D simulations generally predict the impurity transport to be governed by frictional forces for W7-X relevant SOL densities in the range of $1-2 \cdot 10^{19} \mathrm{~m}^{-3}$. In the friction dominated regime, impurities are dragged by the main plasma ions, which flow towards the divertor targets. Therefore, the behavior of the carbon impurity can be used as a good proxy for the main ion species. The observations presented in Sec IIIE show velocities 430 of the same magnitude as the main ion simulation, thereby supporting this conclusion as well.

The comparison of the two diagnostics is presented in Fig 15. Fig[15(a) shows the behavior of both one row of pixels on the camera chip and of one dot representing the selected line of sight magnetically connected to the MPM measurement position, as highlighted in Fig.14(b). The two pixel rows, showing the general tendency of the island chain, have a similar shape, but the measurement with control coil operation (dashed line) shows consistently larger

435 flow velocities along the magnetic field direction (defined as $+v$ ). At the chosen line of sight connected to the MPM position, the difference between both measurements is about $10 \mathrm{~km} / \mathrm{s}$. The value has been confirmed for neighbouring lines of sight. Fig 15(b) shows radial profiles of electron temperature (from a triple Langmuir probe) and parallel flow velocities (from the Mach probe) both along the MPM path. For clarity, the sign of the Mach probe flow has been chosen here in the same way as the CIS flows, on the contrary of the definition in previous publications on Mach 440 probe flows 34, 38, 39. The region which can be used for comparison with CIS is highlighted in yellow. The part outside this region $(\mathrm{R}>6.075 \mathrm{~m})$ should not be considered since it is in the shadow of the divertor, featuring very short connection lengths $(<10 \mathrm{~m})$, so that field lines starting from the probe do not reach the CIS measurement region. Moreover, the electron temperature decay for $\mathrm{R}>6.075 \mathrm{~m}$ shows a tendency similar to the one obtained with no island chain [34. In the highlighted region of interest, the flow velocity in the experiment with control coil operation is 445 about $10 \mathrm{~km} / \mathrm{s}$ higher, well agreeing with the CIS results.

Despite the fact that the absolute velocity values do not agree due to uncertainties in both diagnostics and the complicated mapping between them in the 3D island divertor geometry, the general tendency of increasing flow velocity along the magnetic field direction with the use of control coils $\left(\mathrm{I}_{c c}=2 \mathrm{kA}\right)$ is very robust in both cases. In order to highlight that, the difference has been averaged over the two marked regions of Fig.15, and the results are displayed in

450 Table IV. The last entry of the table shows the average velocity values in the region of interest delimited by the black squared in Fig 14(b), which includes shorter lines of sight and therefore is less affected by line-of-sight integration issues, translating into a good representation for the general edge behavior. This entry confirms that the flow velocity increase is not localized in only one magnetic island. Therefore, the line-of-sight integrated CIS flow pattern confirms that the control coils use affects the entire island chain, adding a significant contribution to the information retrieved 


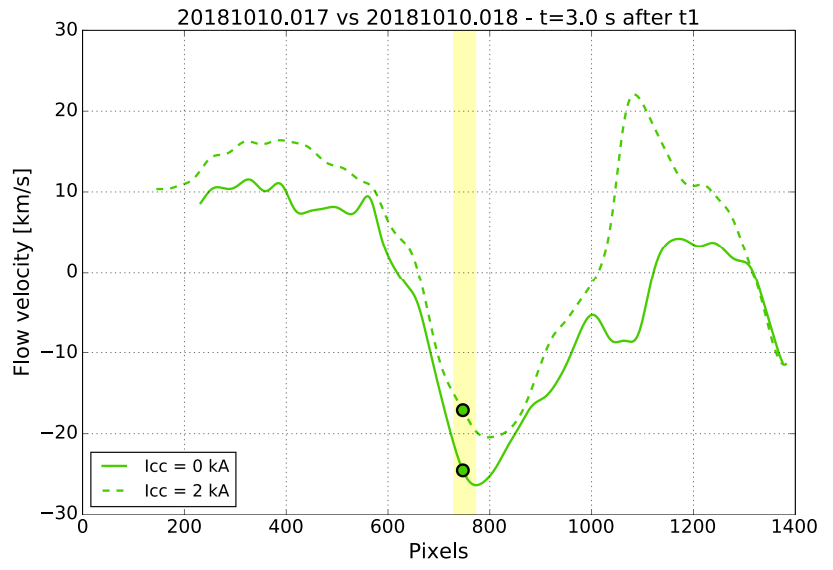

(a)

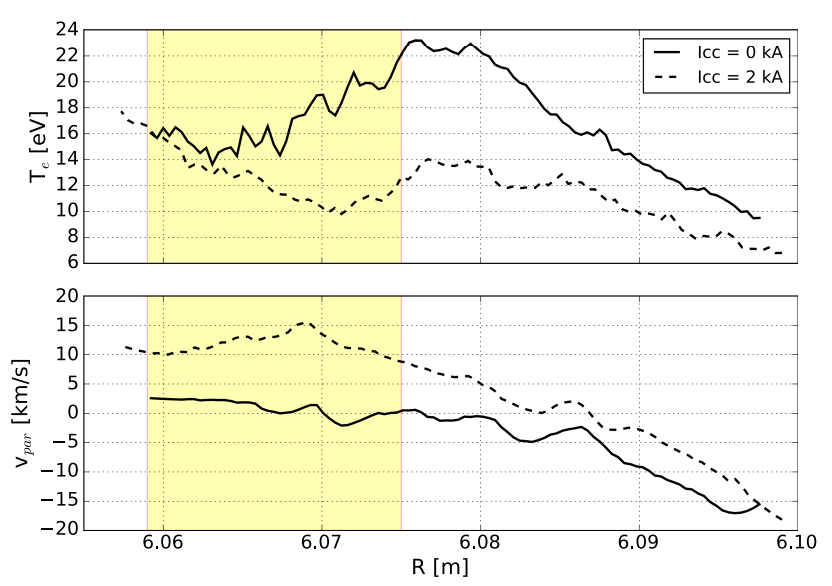

(b)

FIG. 15. Comparison of CIS and Mach probes measurements for discharges without (experiment \#20181010.017, $\mathrm{I}_{c c}=0 \mathrm{kA}$ ) and with (experiment \#20181010.018, $\mathrm{I}_{c c}=2 \mathrm{kA}$ ) control coils. $v>0$ is along the B-field direction. (a) CIS measurement performed with the toroidal system. The line is the flow velocity of one band selected on the camera chip as highlighted in Fig $14(\mathrm{~b})$, the dot represents the selected line of sight. The yellow region is used for the average value displayed in Table IV] (b) Mach probes measurement of electron temperature and parallel velocity. The region of interested for the comparison is highlighted in yellow.

\section{Data trace}

Mach probes

CIS l.o.s.

CIS ROI
Flow velocity difference $[\mathrm{km} / \mathrm{s}]$ $10.92 \pm 2.99$

$7.10 \pm 3.02$

$10.65 \pm 1.69$

TABLE IV. Average values of flow velocity difference with and without control coil use for both CIS and Mach probes. The averages have been calculated in the regions marked in yellow in Fig 15 for the Mach probes and the CIS l.o.s. entries, and in the region of interested delimited by the black square in Fig 14 (b) for the CIS ROI entry.

455 by local probe measurements. The difference in the standard deviations for the two CIS values is to be attributed to the amount of poloidal cross-sections crossed by the lines of sight, which is lower for the CIS ROI case.

\section{CONCLUSIONS}

Two Coherence Imaging Spectroscopy systems have been installed and used at W7-X during the experimental campaign OP1.2. The specific features of W7-X strongly influenced the diagnostic design, specifically regarding the 460 choice of birefringent crystals and narrowband filters. In order to obtain good signal-to-noise ratio, attentive studies on the contrast parameter, with respect to the length of the lines of sight and the presence of additional impurity lines in the filter, have been performed, showing a successful implementation of CIS at W7-X.

The diagnostic has been fully characterized by the use of the new C-Wave tunable laser, which allows a direct calibration of many visible spectral lines. The use of the C-Wave laser improved, at the same time, the flexibility, 465 precision, and stability of the CIS diagnostic, allowing to monitor and measure the system response with little use of simulations and opening up testing possibilities unexplored before.

The importance of a precise calibration source has been highlighted thanks to the comparison with a standard Zn spectral lamp, normally adopted to calibrate C III and He II emission lines. The systems responses have been shown to be sensitive to different wavelength ranges: a $\sim 50 \mathrm{~nm}$ variation towards the green corresponds to a decrease

470 in the induced phase of $\sim 20-22 \%$, implying the necessity of repeating the measurement for any wavelength under investigation, easily doable with the C-Wave laser. A similar wavelength dependency has been identified also for a phase caused by environmental temperature changes. The temperature induced effects appear reproducible and theoretically representable, allowing further developments for a reliable calibration method that will face the challenge of the 30 minutes operation at W7-X without expensive temperature control techniques. An alternative approach 475 to take into account the phase offset introduced by the multiplet structure of the plasma spectral lines have been 
adopted, easing the data analysis process by using an effective wavelength as calibration source. The unambiguous measurement of the relation between $\Delta \Phi$ and $\Delta \lambda$, performed with short range laser scans, allowed to identify the particle flow direction towards the divertor targets in standard plasma parameters.

A comparison with the Multi-Purpose Manipulator Mach probes shows a flow variation of similar tendency and 480 magnitude due to magnetic islands size modification, supporting the reliability of the CIS measurements.

\section{ACKNOWLEDGMENTS}

This work has been carried out within the framework of the EUROfusion Consortium and has received funding from the Euratom research and training program 2014-2018 and 2019-2020 under grant agreement No 633053. The views and opinions expressed herein do not necessarily reflect those of the European Commission. This work was supported 485 in part by the U.S. Department of Energy (DoE) under grants DE-SC0014529 and DE-SC0014210 along with start-up funds from the Physics Department at Auburn University. The publisher, by accepting the article for publication acknowledges, that the United States Government retains a non-exclusive, paid-up, irrevocable, world-wide license to publish or reproduce the published form of this manuscript, or allow others to do so, for United States Government purposes.

490 A special thanks to the technician Marc Gamradt, this diagnostic would have not been successful without his precious and efficient work, Alex Card for the help with the LabView software, and to Marco Krause for the CAD support.

[1] R. C. Isler, N. H. Brooks, W. P. West, and G. D. Porter. Carbon flows in attached divertor plasmas. Physics of Plasmas, 6(5):1837-1842, 1999.

[2] B. LaBombard, J. E. Rice, A. E. Hubbard, J. W. Hughes, M. Greenwald, J. Irby, Y. Lin, B. Lipschultz, E. S. Marmar, C. S. Pitcher, N. Smick, S. M. Wolfe, S. J. Wukitch, and the Alcator Group. Transport-driven Scrape-Off-Layer flows and the boundary conditions imposed at the magnetic separatrix in a tokamak plasma. Nuclear Fusion, 44(10), 2004.

[3] M. Kobayashi, Y. Feng, S. Morita, S. Masuzaki, N. Ezumi, T. Kobayashi, M. B. Chowdhuri, H. Yamada, T. Morisaki, N. Ohyabu, M. Goto, I. Yamada, K. Narihara, A. Komori, O. Motojima, and LHD Experiment Group. Transport characteristics in the stochastic magnetic boundary of LHD: Magnetic field topology and its impact on divertor physics and impurity transport. Fusion Science and Technology, 58, 2010.

[4] M. Kobayashi, S. Morita, and M. Goto. 2D distribution of hydrogen/impurity radiation and flow formation in stochastic layer during detachment transition in LHD. Nuclear Materials and Energy, 12:1043 - 1048, 2017. Proceedings of the 22nd International Conference on Plasma Surface Interactions 2016, 22nd PSI.

505 [5] J. Howard. Novel scanning interferometer for two-dimensional plasma density measurements. Review of Scientific Instruments, 61:1086-1094, 031990.

[6] J. Howard. Coherence imaging spectro-polarimetry for magnetic fusion diagnostics. Journal of Physics B: Atomic, Molecular nad Optical Physics, 43(144010), 2010.

[7] J. Howard, C. Michael, F. Glass, and A. Danielsson. Time-resolved two-dimensional plasma spectroscopy using coherenceimaging techniques. Plasma Physics and Controlled Fusion, 45:1143-1166, 2003.

[8] J. Howard, A. Diallo, M. Creese, B. D. Blackwell, S. L. Allen, R. M. Ellis, G. D. Porter, W. Meyer, M. E. Fenstermacher, N. H. Brooks, M. E. Van Zeeland, and R. L. Boivin. Doppler coherence imaging and tomography of flows in tokamak plasmas (invited). Review of Scientific Instruments, 81(10):10E528, 2010.

[9] S. A. Silburn, J. R. Harrison, J. Howard, K. J. Gibson, H. Meyer, C. A. Michael, and R. M. Sharples. Coherence

515 imaging of scrape-off-layer and divertor impurity flows in the Mega Amp Spherical Tokamak (invited). Review of Scientific Instruments, 85(11):11D703, 2014.

[10] J. Howard, A. Diallo, M. Creese, S. L. Allen, R. M. Ellis, W. Meyer, M. E. Fenstermarcher, G. D. Porter, H. Brooks, M. E. Van Zeeland, and R. L. Boivin. Coherence imaging of flows in the DIII-D divertor. Contributions to Plasma Physics, 51:194-200, 032011.

520 [11] C. M. Samuell, G. D. Porter, W. H. Meyer, T. D. Rognlien, S. L. Allen, A. Briesemeister, A. G. Mclean, L. Zeng, A. E. Jaervinen, and J. Howard. 2D imaging of helium ion velocity in the DIII-D divertor. Physics of Plasma, 25(056110), 2018.

[12] C. M. Samuell, S. L. Allen, W. H. Meyer, R. C. Isler, A. Briesemeister, R. S. Wilcox, C. J. Lasnier, A. G. Mclean, and J. Howard. Verification of Doppler coherence imaging for 2D ion velocity measurements on DIII-D. Review of Scientific Instruments, 89(093502), 2018.

525 [13] D. Gradic, O. P. Ford, A. Burckhart, F. Effenberg, H. Frerichs, R. König, T. Lunt, V. Perseo, R. C. Wolf, ASDEX Upgrade Team, W7-X Team, and Eurofusion MST1 Team. Doppler coherence imaging of divertor and SOL flows in ASDEX upgrade and Wendelstein 7-X. Plasma Physics and Controlled Fusion, 60(084007), 2018.

[14] V. Perseo, R. König, C. Biedermann, O. Ford, D. Gradic, M. Krychowiak, G. Kocsis, D. Ennis, D. Maurer, T. Sunn Ped- 
ersen, and the W7-X Team. Coherence Imaging Spectroscopy systems on Wendelstein 7-X for studies of island divertor plasma behavior. 44th EPS Conference on Plasma Physics, 2017.

[15] S. L. Allen, C. M. Samuell, W. H. Meyer, and J. Howard. Laser calibration of the DIII-D coherence imaging system. Review of Scientific Instruments, 89, 2018.

[16] D. Gradic, V. Perseo, R. König, and D. Ennis. A new calibration implementation for Doppler Coherence Imaging Spectroscopy. Fusion Engineering and Design, 2019.

535 [17] V. Perseo, F. Effenberg, D. Gradic, R. König, O. P. Ford, R. Felix, D. A. Ennis, O. Schmitz, T. Sunn Pedersen, and the W7-X Team. Direct measurements of counter-streaming flows in a low-shear stellarator magnetic island topology. Nuclear Fusion, 59(12):124003, 2019.

[18] H.-S. Bosch, R. Brakel, T. Braeuer, V. Bykov, P. van Eeten, J.-H. Feist, F. Füllenbach, M. Gasparotto, H. Grote, T. Klinger, H. Laqua, M. Nagel, D. Naujoks, M. Otte, K. Risse, T. Rummel, J. Schacht, A. Spring, T. Sunn Pedersen, R. Vilbrandt,

540 Wegener L., A. Werner, R. C. Wolf, J. Baldzuhn, C. Biedermann, H. Braune, R. Burhenn, M. Hirsch, U. Höfel, J. Knauer, P. Kornejew, S. Marsen, T. Stange, H. Trimino Mora, and the W7-X Team. Final integration, commissioning and start of the Wendelstein 7-X stellarator operation. Nuclear Fusion, 57(11):116015, 2017.

[19] G. Kocsis, T. Baross, C. Biedermann, G. Bodnár, G. Cseh, T. Ilkei, R. König, M. Otte, T. Szabolics, T. Szepesi, and S. Zoletnik. Overview video diagnostics for the W7-X stellarator. Fusion Engineering and Design, 96-97:808 - 811, 2015. Proceedings of the 28th Symposium On Fusion Technology (SOFT-28).

[20] G. Kocsis, A. Alonso, C. Biedermann, G. Cseh, A. Dinklage, O. Grulke, M. Jakubowski, R. König, M. Krychowiak, M. Otte, T. Sunn Perdersen, T. Szepesi, U. Wenzel, P. Xanthopoulos, S. Zoletnik, and the W7-X Team. Characterization of edge filamentary structures in the 3D geometry of Wendelstein 7-X limiter plasmas. 44th EPS Conference on Plasma Physics, 2017.

550 [21] M. Jakubowski, P. Drewelow, J. Fellinger, A. Puig Sitjes, G. Wurden, A. Ali, C. Biedermann, B. Cannas, D. Chauvin, M. Gamradt, H. Greve, Y. Gao, D. Hathiramani, R. König, A. Lorenz, V. Moncada, H. Niemann, T. T. Ngo, F. Pisano, T. Sunn Pedersen, and the W7-X Team. Infrared imaging systems for wall protection in the W7-X stellarator (invited). Review of Scientific Instruments, 89(10):10E116, 2018.

[22] Scott Alan Silburn. A Doppler Coherence Imaging diagnostic for the Mega-Amp Spherical Tokamak. PhD thesis, Durham University, 092014.

[23] S. L. Allen, W. H. Meyer, G. D. Porter, and J. Howard. Carbon ion flow measurements in DIII-D divertors by coherence imaging. 21st Plasma Surface Interactions Meeting, 052014.

[24] R. Lester, Y. Zhai, C. Corr, and J. Howard. Coherence imaging for ion temperature and flow measurements in a lowtemperature helicon plasma source. Plasma Sources Science and Technology, 25:015025, 2016.

560 [25] Romana Lester. Doppler coherence imaging of ion flows and temperatures in the MAGPIE helicon plasma. PhD thesis, ANU College of Science, The Australian National University, 2019.

[26] F. E. Veiras, L. I. Perez, and M. T. Garea. Phase shift formulas in uniaxial media: an application to waveplates. Applied Optics, 49(15), 052010.

[27] J. Sperling and K. Hens. Tunable laser light sources advance nanophotonics research. Photonics Media, 2018.

565 [28] M. Endler, J. Fellinger, H. Hölbe, T. Sunn Pedersen, S. Bozhenkov, J. Geiger, M. Grahl, and the W7-X team. Managing leading edges during assembly of the Wendelstein 7-X divertor. Plasma Physics and Controlled Fusion, 61(2):025004, nov 2018.

[29] M. Krychowiak, A. Adnan, A. Alonso, T. Andreeva, J. Baldzuhn, T. Barbui, M. Beurskens, W. Biel, C. Biedermann, B. D. Blackwell, H. S. Bosch, S. Bozhenkov, R. Brakel, T. Bräuer, B. Brotas de Carvalho, R. Burhenn, B. Buttenschön, A. Cappa, G. Cseh, A. Czarnecka, A. Dinklage, P. Drews, A. Dzikowicka, F. Effenberg, M. Endler, V. Erckmann, T. Estrada, O. Ford, T. Fornal, H. Frerichs, G. Fuchert, J. Geiger, O. Grulke, J. H. Harris, H. J. Hartfuß, D. Hartmann, D. Hathiramani, M. Hirsch, U. Höfel, S. Jabłoński, M. W. Jakubowski, J. Kaczmarczyk, T. Klinger, S. Klose, J. Knauer, G. Kocsis, R. König, P. Kornejew, A. Krämer-Flecken, N. Krawczyk, T. Kremeyer, I. Ksiażek, M. Kubkowska, A. Langenberg, H. P. Laqua, M. Laux, S. Lazerson, Y. Liang, S. C. Liu, A. Lorenz, A. O. Marchuk, S. Marsen, V. Moncada, D. Naujoks, H. Neilson, O. Neubauer, U. Neuner, H. Niemann, J. W. Oosterbeek, M. Otte, N. Pablant, E. Pasch, T. Sunn Pedersen, F. Pisano, K. Rahbarnia, L. Ryć, O. Schmitz, S. Schmuck, W. Schneider, T. Schröder, H. Schuhmacher, B. Schweer, B. Standley, T. Stange, L. Stephey, J. Svensson, T. Szabolics, T. Szepesi, H. Thomsen, J.-M. Travere, H. Trimino Mora, H. Tsuchiya, G. M. Weir, U. Wenzel, A. Werner, B. Wiegel, T. Windisch, R. Wolf, G. A. Wurden, D. Zhang, A. Zimbal, and S. Zoletnik. Overview of diagnostic performance and results for the first operation phase in Wendelstein 7-X (invited). Review of Scientific Instruments, 87(11):11D304, 2016.

[30] R. Brakel, A. Dinklage, J. Fellinger, G. Fuchert, H. Grote, R. König, H. Laqua, T. Stange, O. Volzke, G. Schlisio, U. Wenzel, D. Zhang, A. Goriaev, T. Wauters, S. Brezinsek, V. E. Moiseenko, A. A. Belitskii, and the W7-X team. Strategy and optimisation of wall conditioning at the Wendelstein 7-X stellarator. Preprint: 2018 IAEA Fusion Energy Confinement (India), (EX/P8-17), 2018.

585 [31] C. M. Samuell, S. L. Allen, W. H. Meyer, and J. Howard. Absolute calibration of Doppler coherence imaging velocity images. Journal of Instrumentation, 12, 082017.

[32] R. C. Wolf, A. Alonso, S. Äkäslompolo, J. Baldzuhn, M. Beurskens, C. D. Beidler, C. Biedermann, H.-S. Bosch, S. Bozhenkov, R. Brakel, H. Braune, S. Brezinsek, K.-J. Brunner, H. Damm, A. Dinklage, P. Drewelow, F. Effenberg, Y. Feng, O. Ford, G. Fuchert, Y. Gao, J. Geiger, O. Grulke, N. Harder, D. Hartmann, P. Helander, B. Heinemann, M. Hirsch, U. Höfel, C. Hopf, K. Ida, M. Isobe, M. W. Jakubowski, Y. O. Kazakov, C. Killer, T. Klinger, J. Knauer, R. König, M. Krychowiak, A. Langenberg, H. P. Laqua, S. Lazerson, P. McNeely, S. Marsen, N. Marushchenko, R. Nocentini, K. Ogawa, G. Orozco, M. Osakabe, M. Otte, N. Pablant, E. Pasch, A. Pavone, M. Porkolab, A. Puig Sitjes, 
K. Rahbarnia, R. Riedl, N. Rust, E. Scott, J. Schilling, R. Schroeder, T. Stange, A. von Stechow, E. Strumberger, T. Sunn Pedersen, J. Svensson, H. Thomson, Y. Turkin, L. Vano, T. Wauters, G. Wurden, M. Yoshinuma, M. Zanini, and D. Zhang. Performance of Wendelstein 7-X stellarator plasmas during the first divertor operation phase. Physics of Plasmas, 26(8):082504, 2019.

[33] H. Hölbe. Control of the magnetic topology and plasma exhaust in the edge region of Wendelstein 7-X: a numerical study. PhD thesis, Max Planck Institute for Plasma Physics, 2015.

[34] C. Killer, O. Grulke, P. Drews, Y. Gao, M. Jakubowski, A. Knieps, D. Nicolai, H. Niemann, A. Puig Sitjes, G. Satheeswaran, and the W7-X Team. Characterization of the W7-X scrape-off layer using reciprocating probes. Nuclear Fusion, 59(8):086013, jun 2019.

[35] S. A. Barbui, T. Bozhenkov, F. Effenberg, C. Favreau, E. Flom, G. Fuchert, P. Drews, K. Hammond, M. Jakubowski, C. Killer, R. König, M. Krychowiak, S. Loch, E. Munos Burgos, J. Pasch, E. R. Schmitz, O. Scott, M. Vecsei, S. Zoletnik, and the W7-X team. The He/Ne beam diagnostic for line-ratio spectroscopy in the island divertor of Wendelstein 7-X. Journal of Instrumentation, 14, 2019.

[36] P. C. Stangeby. The plasma boundary of magnetic fusion devices. IoP, 2000.

[37] D. Nicolai, V. Borsuk, P. Drews, O. Grulke, K. P. Hollfeld, T. Krings, Y. Liang, Ch. Linsmeier, O. Neubauer, G. Satheeswaran, B. Schweer, and G. Offermanns. A multi-purpose manipulator system for W7-X as user facility for plasma edge investigation. Fusion Engineering and Design, 123:960 - 964, 2017. Proceedings of the 29th Symposium on Fusion Technology (SOFT-29) Prague, Czech Republic, September 5-9, 2016.

[38] J. Cai, Y. Liang, C. Killer, S. Liu, A. Hiller, A. Knieps, B. Schweer, D. Höschen, D. Nicolai, G. Offermanns, G. Satheeswaran, M. Henkel, K. Hollfeld, O. Grulke, P. Drews, T. Krings, and Y. Li. A new multi-channel Mach probe measuring the radial ion flow velocity profile in the boundary plasma of the W7-X stellarator. Review of Scientific Instruments, 90(3):033502, 2019.

615 [39] C. Killer, Y. Gao, V. Perseo, L. Rudischhauser, K. Hammond, B. Buttenschön, T. Barbui, B. D. Blackwell, K. J. Brunner, P. Drews, M. B. S. Endler, J. Geiger, O. Grulke, M. Jakubowski, S. Klose, J. P. Knauer, A. Knieps, R. König, Y. Li, U. Neuner, H. Niemann, M. Otte, J. Schilling, A. Puig Sitjes, K. Rahbarnia, T. Stange, and the W7-X Team. Effect of toroidal plasma currents on the Wendelstein 7-X Scrape-Off Layer. Plasma Physics and Controlled Fusion, 2019.

[40] S. A. Bozhenkov, M. Otte, C. Biedermann, M. Jakubowski, S. A. Lazerson, T. Sunn Pedersen, R. C. Wolf, and the W7-X team. Measurements and correction of the 1/1 error field in Wendelstein 7-X. Nuclear Fusion, 59(2):026004, dec 2018.

[41] Y. Feng, F. Sardei, P. Grigull, K. McCormick, J. Kisslinger, and D. Reiter. Physics of island divertors as highlighted by the example of W7-AS. Nuclear Fusion, 46:807-819, 2006. 